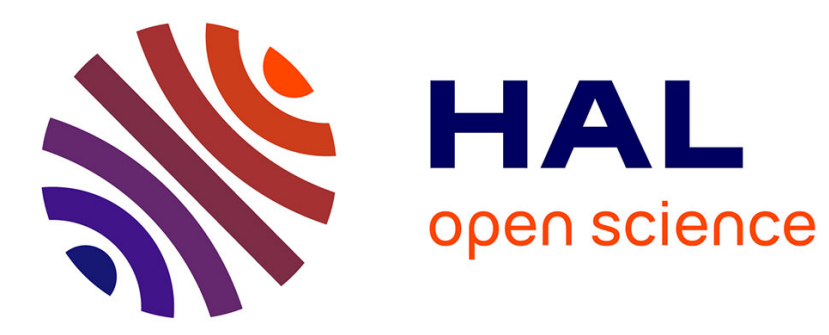

\title{
Ammonia Clathrate Hydrate As Seen from Grand Canonical Monte Carlo Simulations
}

Balázs Fábián, Sylvain Picaud, Pál Jedlovszky, Aurélie Guilbert-Lepoutre, Olivier Mousis

\section{> To cite this version:}

Balázs Fábián, Sylvain Picaud, Pál Jedlovszky, Aurélie Guilbert-Lepoutre, Olivier Mousis. Ammonia Clathrate Hydrate As Seen from Grand Canonical Monte Carlo Simulations. ACS Earth and Space Chemistry, 2018, 10.1021/acs earth space chem.7b00133 . hal-03547559

\author{
HAL Id: hal-03547559 \\ https://hal.science/hal-03547559
}

Submitted on 28 Jan 2022

HAL is a multi-disciplinary open access archive for the deposit and dissemination of scientific research documents, whether they are published or not. The documents may come from teaching and research institutions in France or abroad, or from public or private research centers.
L'archive ouverte pluridisciplinaire HAL, est destinée au dépôt et à la diffusion de documents scientifiques de niveau recherche, publiés ou non, émanant des établissements d'enseignement et de recherche français ou étrangers, des laboratoires publics ou privés. 


\title{
Ammonia Clathrate Hydrate As Seen from Grand Canonical Monte Carlo Simulations
}

\author{
Balázs Fábián ${ }^{1,2}$, Sylvain Picaud*1*, Pál Jedlovszky ${ }^{3}$, Aurélie \\ Guilbert-Lepoutre $^{1}$, Olivier Mousis ${ }^{4}$
}

\author{
${ }^{1}$ Institut UTINAM, UMR 6213 CNRS/Université Bourgogne Franche-Comté, \\ 16 route de Gray, $F-25030$ Besançon Cedex, France \\ ${ }^{2}$ Department of Inorganic and Analytical Chemistry, Budapest University of \\ Technology and Economics, Szt. Gellért tér 4, H-1111 Budapest, Hungary \\ ${ }^{3}$ Department of Chemistry, Eszterházy Károly University, Leányka utca 6, H- \\ 3300 Eger, Hungary \\ ${ }^{4}$ Aix Marseille Université, CNRS, LAM (Laboratoire d'Astrophysique de \\ Marseille), UMR 7326, F-13388 Marseille, France
}

Running title: Grand Canonical Monte Carlo simulation of $\mathrm{NH}_{3}$ clathrate keywords : Clathrate, Ammonia, Grand Canonical Monte Carlo, Simulations, Planetary Science. e-mail: sylvain.picaud@univ-fcomte.fr (SP), jedlovszky.pal@uni-eszterhazy (PJ)

\begin{abstract}
In this paper, the trapping of ammonia molecules into a clathrate structure has been investigated by means of Grand Canonical Monte Carlo simulations, performed at three different temperatures $(100,150$ and $180 \mathrm{~K})$ relevant for the astrophysics environments. The results show that ammonia clathrate of structure I is stable at partial filling, irrespective of the temperature investigated here. It could also be metastable in a chemical potential (pressure) range that corresponds to a maximum of 8 ammonia molecules per unit cell, i.e., to the full occupancy of the clathrate structure at very low temperature. However, at higher chemical potential values, partial dissolution of the clathrate is evidenced, concomitant with its transformation to low-density amorphous ice at 150 and $180 \mathrm{~K}$. In the clathrate stability regime, it is shown that ammonia molecules can also displace water molecules and become incorporated into the water lattice, which results in the progressive destabilization of the clathrate lattice with increasing number of trapped ammonia molecules. Our results point out the subtle interplay between the various
\end{abstract}


environmental conditions (temperature, partial pressure of ammonia) on the stability of the clathrate phase in various planetary environments. 


\section{Introduction}

Ammonia is a common molecule in the universe. Its presence has widely been evidenced in the interstellar medium [1] as well as in the primitive matter of the solar system (meteorites, comets) and in the atmospheres of Jupiter and Saturn, the two gas giants orbiting the Sun.[2] Ammonia is also assumed to be the main source of the molecular nitrogen dominating Titan'atmosphere.[3]. The ammonia content and ammonia/water ratio seems also to be critical in search for amino acids, which are basic life molecules.[4]

Solar system bodies may have preserved ammonia in the form of ammonium silicates or in ice. The presence of ammonia hydrates has thus been hypothesized in the Saturn's largest moon Titan,[5,6] and also evidenced on the surfaces of the minor planets Charon [7] and Orcus [8] via observations in the near-infrared range. The presence of ammonia hydrate, together with crystalline water ice, has been invoked as an evidence for past or, even present, cryovolcanism on the Kuiper Belt objects.[9]

On the other hand, ammonia is suspected to modify the stability regions of the solid ice and methane clathrate phases on various moons in the Solar System.[10] Indeed, it has been experimentally shown that the hydrophilic behavior of ammonia does not disqualify this properly sized molecule from being incorporated into clathrates, together with a more hydrophobic guest that is known to easily form stable clathrates.[10] Ammonia may even form clathrate hydrates between 100 and $150 \mathrm{~K}$, with only ammonia as guest,[10] as shown by some peak positions in Xray diffraction measurements that can be unambiguously assigned to the ammonia clathrate hydrate with cubic structure I (sI).[10] The stability of this sI clathrate structure, together with a detailed analysis of the hydrogen bonding between water and ammonia molecules has then be theoretically investigated by molecular dynamics (MD) simulations, showing that the pure $\mathrm{sI} \mathrm{NH}_{3}$ clathrate decomposes in the simulations between 220 and $240 \mathrm{~K}$, depending on the water force field used.[11]

Indeed, considering both the paucity of experimental data on the ammonia clathrate itself and the lack of a fundamental understanding of how the $\mathrm{NH}_{3}$ molecule may affect the phase behavior of other clathrates, computer simulation appears to be an ideal tool for providing the missing insight at the atomistic level. Thus, MD simulations have been performed to characterize not only the structure and the stability of pure $\mathrm{NH}_{3}$, but also of binary $\mathrm{NH}_{3}+\mathrm{THF}$ (tetrahydrofuran) and $\mathrm{CH}_{4}+\mathrm{NH}_{3}$ clathrates from 100 to $240 \mathrm{~K}$, in close relation with the 
corresponding experimental work.[10,11]. However, such simulations, performed on the isothermal-isobaric $(N, p, T)$ ensemble, suffer from their dependence on the initial conditions, especially regarding the clathrate composition that has to be fixed in the calculations. Moreover, it is sometimes difficult with MD to know if the simulated system corresponds to a real equilibrium structure or if it is stuck in a metastable state, even when performing very long simulation runs. Because the abundance of the trapped gases in the hosting cages of the clathrates under consideration is one of the most important data sought, calculations performed on the grand canonical ensemble, where the number of guest molecules is not fixed in the simulations, may appear to be much more appropriate. In simulations performed on the grand canonical ensemble, such as grand canonical Monte Carlo (GCMC) [12,13] simulations, the chemical potential rather than the number of the molecules is fixed. Thus, performing a set of GCMC simulations, in which the chemical potential of the molecules in contact with the absorbent system is systematically varied, the occupancy isotherm, i.e., the number of enclathrated molecules as a function of their chemical potential can be conveniently calculated. Surprisingly this method has only scarcely been applied to clathrate systems since the pioneering work of Tanaka et al. $[14,15]$, although it has proven to be very efficient for directly calculating the composition of methane [16,17], hydrogen[18] binary hydrogen/THF [19] and binary $\mathrm{CO} / \mathrm{N}_{2}$ [20] clathrates under various thermodynamic conditions.

In this paper, we present the first GCMC simulation study of the trapping of $\mathrm{NH}_{3}$ molecules in the cages of a clathrate hydrate of structure I in the very low temperature range of [100 - 180] K, which was also experimentally investigated.[10] Besides calculating the trapping isotherm, the detailed analysis of the clathrate structure is also performed at various ammonia abundances. In Sec. 2 details of the calculations performed are given. The obtained results concerning the occupancy isotherm and properties of the resulting systems are presented and discussed in detail in Sec. 3. Finally, in Sec. 4 the main conclusions of this study are summarized.

\section{Grand Canonical Monte Carlo simulations}

The trapping of $\mathrm{NH}_{3}$ molecules in the clathrate hydrate lattice has been investigated by performing a set of Monte Carlo simulations on the grand canonical $(\mu, V, T)$ ensemble at three temperatures, namely 100, 150 and $180 \mathrm{~K}$. Simulations have first been performed with a rigid clathrate, i.e., a system where the water molecules have been fixed at the equilibrium positions of 
a clathrate of structure I (sI), with fixed orientations. While the number of water molecules in the simulation box has been kept constant, the number of ammonia molecules trapped by the rigid network of water has been calculated by increasing the chemical potential, $\mu$, of the $\mathrm{NH}_{3}$ molecules from values corresponding to empty clathrate lattice to values corresponding to twice the number of cages. The isotherms were obtained as the average number of $\mathrm{NH}_{3}$ molecules, $\langle N\rangle$, in the simulation box as a function of the chemical potential, and will be referred to as occupancy isotherms because the number of enclathrated ammonia molecules is calculated.[16] Simulations have been performed with simulation boxes based on both $2 \times 2 \times 2$ and $3 \times 3 \times 3$ replicas of the sI unit cell, taken from Takeuchi et al.,[21] to test the influence of the size of the simulation box in the calculated isotherms. The $2 \times 2 \times 2$ replica of the unit cell of sI clathrate consisted of a total of 368 water molecules forming a network of 48 large and 16 small cages. The edge length of this cubic box was $24.06 \AA$ in every direction, in agreement with the periodicity of the clathrate lattice. The larger, $3 \times 3 \times 3$, replica of the unit cell corresponded to a network of 1242 water molecules forming 162 large and 54 small cages in a cubic simulation box with the edge length equal to $36.09 \AA$.

Then, the GCMC method has also been applied to a flexible clathrate model, in which rotational and translational degrees of freedom of the water molecules have also been considered. However, no chemical potential value has been assigned to the water molecules, and thus insertion/deletion steps have not been considered for water molecules in the GCMC calculations. Nevertheless, this treatment has resulted in a large increase of the corresponding computational cost and, as a consequence, simulations with flexible clathrates have only been performed with the $2 \times 2 \times 2$ simulation.

To facilitate the comparison of our results with those of the molecular dynamics simulations of Alavi et al.,[11] water molecules have been described by the TIP4P/Ice potential.[22] This potential was shown to reproduce more accurately the decomposition temperature of methane hydrate while also giving better predictions regarding the lattice constants, as compared to the TIP4P model.[23] Moreover, the fact that TIP4P/Ice tends to form less hydrogen bonds with the $\mathrm{NH}_{3}$ molecules increases the likelihood of the clathrate being stable in the temperature range of the simulations.[11] The $\mathrm{NH}_{3}$ molecules have been represented by the OPLS force-field of Rizzo and Jorgensen,[24] again to ensure comparability. Based on these models, the potential energy has been calculated as the sum of the pairwise interaction energies 
between molecules, where the interaction energy between two molecules is defined as the sum of the Lennard-Jones and Coulomb contributions of all pairs of their interaction sites. For the potential models used here, these interaction sites are located at the atomic positions, with the exception of the TIP4P/Ice model, which contains also a non-atomic site slightly displaced from the $\mathrm{O}$ atom, along the water molecular axis.[22]

All interactions have been truncated to zero beyond the distance of $12.03 \AA$ and $18.04 \AA$ for the small and the large simulation box, respectively. The long range contribution of the electrostatic interaction has been accounted for by the reaction field correction method $[25,26]$ under conducting boundary conditions. The interaction and geometric parameters of the employed models are given in Table 1.

Monte Carlo simulations have been performed using the MMC code [30] that has been widely applied for studying the adsorption process of a number of atmospherically relevant molecules at the surface of ice,[28-36] showing a fair agreement with the corresponding experimental results when available. In every Monte Carlo step, a translational and rotational move was attempted with $50 \%$ probability on a randomly chosen molecule, along a randomly chosen axis parallel to one of the edges of the simulation box. The maximum value of these translations and rotations did not exceed $0.25 \AA$ and $15^{\circ}$, respectively, and the acceptance or rejection of these steps was evaluated based on the standard Metropolis criterion.[37]. Insertion and deletion of the guest $\mathrm{NH}_{3}$ molecules was performed with the remaining $25 \%-25 \%$ probabilities, using the cavity biased technique of Mezei.[38,39] According to this method, the guest molecule insertions were only attempted into empty cavities having a minimum radius of $2.5 \AA$ A Cavities were identified along a $100 \times 100 \times 100$ grid in the basic simulation box, which was regenerated after every $10^{6}$ Monte Carlo steps. The Monte Carlo acceptance rule was modified accordingly, in order to eliminate the bias introduced this way.[38,39] The systems were equilibrated by performing $5 \times 10^{8}$ Monte Carlo steps. For each system, the equilibration phase was followed by the production phase during which a total of 5000 configurations with a sampling interval of $10^{5}$ steps between samples were collected for further analyses. 


\section{Results and Discussion}

\subsection{Occupancy Isotherm}

The occupancy isotherms calculated for rigid and flexible clathrate structures, i.e., the average number of ammonia molecules enclathrated as a function of the ammonia chemical potential, are given in Figure 1, while several equilibrium snapshots taken out from the simulations are shown in Figure 2.
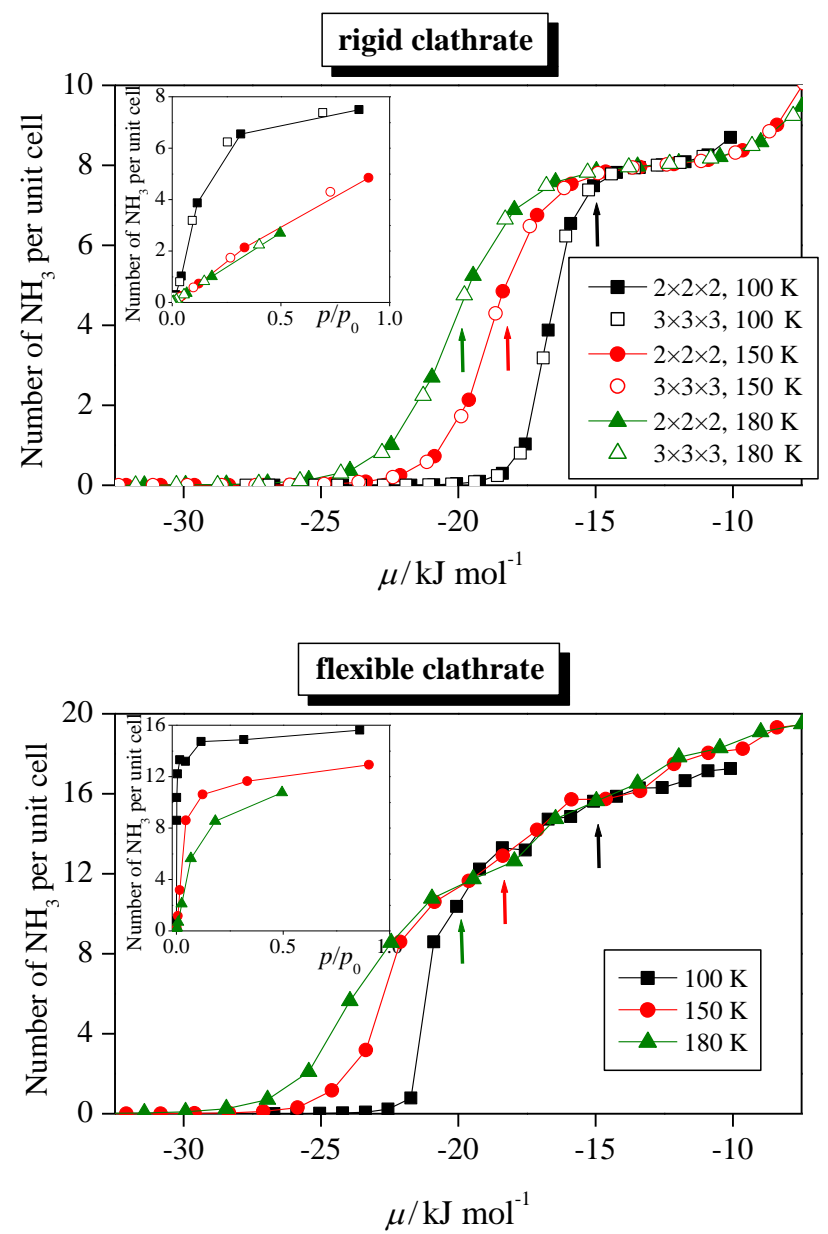

Fig. 1. Occupancy isotherm of $\mathrm{NH}_{3}$ in a (a) rigid (top panel) and (b) flexible (bottom panel) clathrate hydrate, as obtained from our GCMC simulations at $100 \mathrm{~K}$ (black squares), $150 \mathrm{~K}$ (red circles) and $180 \mathrm{~K}$ (green triangles). Filled and empty symbols correspond to $2 \times 2 \times 2$ and $3 \times 3 \times 3$ clathrate units in the basic box. The insets show the occupancy isotherms calculated as a function of the relative pressure $\mathrm{p}_{\mathrm{rel}}=\mathrm{p} / \mathrm{p}_{0}$. The lines connecting the points are just guides to the eye. The arrows indicate the chemical potential values corresponding to the point of condensation of $\mathrm{NH}_{3}$. Note that, for the ordinate axis, we divide the number of ammonia molecules in the simulation box by the number of unit cells of the rigid clathrate, as an arbitrary normalization. 
First, it has to be mentioned that in the temperature range investigated here the temperature has very little influence on the behavior of the ammonia occupancy isotherm. Indeed, irrespective of the temperature, the occupancy isotherm is characterized first by a continuous exponential increase at low chemical potential values, corresponding to the progressive filling of the clathrate cages by the ammonia molecules. Then, above around $-16 \mathrm{~kJ} / \mathrm{mol}$, the isotherm for the rigid clathrate (Figure 1a) reaches a rather long plateau covering a roughly $6-8 \mathrm{~kJ} / \mathrm{mol}$ wide range of chemical potentials. For this range of chemical potentials, the clathrate appears saturated and changes in the chemical potential values result only in a rather small increase of the number of enclathrated molecules. In the corresponding system, 8 ammonia molecules are trapped per unit cell of the simulation box, i.e., there is, on average, one ammonia molecule per cage in the clathrate. Finally, at a value of about $-9 \mathrm{~kJ} / \mathrm{mol}$, the isotherm increases again, corresponding to the possible trapping of additional ammonia molecules in the clathrate cages at very large chemical potential values, i.e., at very high pressures. Moreover, the comparison between the isotherms calculated for the rigid clathrate by considering $2 \times 2 \times 2$ and $3 \times 3 \times 3$ simulation boxes (Figure 1a) suggests that the effect of the box size on the results is negligibly weak, since the corresponding occupancy isotherms are almost perfectly superimposed. This indicates that simulations performed by considering the small, $2 \times 2 \times 2$ simulation box can provide results of reasonable quality concerning the cage occupancy calculations at a significantly lower computational cost than when considering the larger, $3 \times 3 \times 3$ box.

The comparison between the ammonia occupancy isotherms simulated for flexible clathrate structures at 100, 150 and $180 \mathrm{~K}$ (Figure 1b) and those given in Figure 1a for rigid clathrates immediately shows the influence of the cage flexibility on the trapping of the ammonia molecules inside the clathrate. Indeed, not only the chemical potential value at which the filling of the clathrate cages starts is lower for flexible than for rigid structures, but also the global shape of the isotherm looks different. Indeed, the isotherm for flexible clathrate is characterized by an almost continuous rise with increasing chemical potential, and no plateau is evidenced contrarily to the rigid clathrate situation. Overall, for any given chemical potential and temperature value, the occupancy of the flexible clathrate is higher than that of the rigid one. More surprisingly, the number of ammonia molecules that can be inserted in the simulation box rapidly exceeds the number of cages upon the increase of the chemical potential, which rises a question about the 
stability of the clathrate in such conditions and, as a consequence, about the exact nature of the corresponding system.

Another way to represent the obtained occupancy isotherms is to convert the chemical potential to relative pressure $\mathrm{p}_{\mathrm{rel}}=\mathrm{p} / \mathrm{p}_{0}$, i.e., the pressure of the system, $\mathrm{p}$, normalized by that of the saturated vapor, $\mathrm{p}_{0}$. Since the bulk vapor phase of the system is practically empty in every case, the value of the absolute pressure, p, can hardly be evaluated in the simulations. However, the relative pressure can simply be calculated as [40]

$$
p_{\text {rel }}=\frac{p}{p_{0}}=\frac{\exp \left(\mu / k_{\mathrm{B}} T\right)}{\exp \left(\mu_{0} / k_{\mathrm{B}} T\right)}
$$

where $k_{\mathrm{B}}$ stands for the Boltzmann constant, and $\mu_{\mathrm{o}}$ is the chemical potential value corresponding to the point of condensation. To determine this $\mu_{0}$ value, we have performed a new set of GCMC simulations by considering only the $\mathrm{NH}_{3}$ molecules in a box containing no water molecule and having the same volume as the box used for the clathrate simulation. In this case, there is no absorption/incorporation (as there is no clathrate), but only condensation of ammonia at a certain point, corresponding to the chemical potential value $\mu_{0}$. The occupancy isotherms as a function of $p_{\text {rel }}$ are shown in the inset of Fig. 1, and the corresponding data are included in Tables S1 to S6 of the Supporting Information. Note that the $\mathrm{p}_{0}$ value has been determined here only as a reference value for converting chemical potentials to relative pressures. Moreover, because Eq. (3) is only valid up to the point of condensation, the conversion from $\mu$ to $\mathrm{p}_{\text {rel }}$ has not been done above the value $\mathrm{p} / \mathrm{p}_{0}=1$.

Note that the value of $\mathrm{p}_{0}$ could, in principle, be obtained from the Antoine's equation

$$
\log _{10} p_{0}=A-\frac{B}{T+C}
$$

where the temperature is in $\mathrm{K}$ and the pressure in bar. To the best of our knowledge, the Antoine's coefficients for ammonia are unfortunately not known below $164 \mathrm{~K}$. However, in the [164 - 240] $\mathrm{K}$ range, their values are $\mathrm{A}=3.18757, \mathrm{~B}=506.713 \mathrm{~K}, \mathrm{C}=-80.78 \mathrm{~K}$ [41], which gives $\mathrm{p}_{0}=0.012$ bar at $180 \mathrm{~K}$. By contrast, $\mathrm{p}_{0}$ values at 100 and $150 \mathrm{~K}$ cannot be calculated, 
which prevents any reasonable comparison with actual physical conditions for these very low temperatures.

(a)
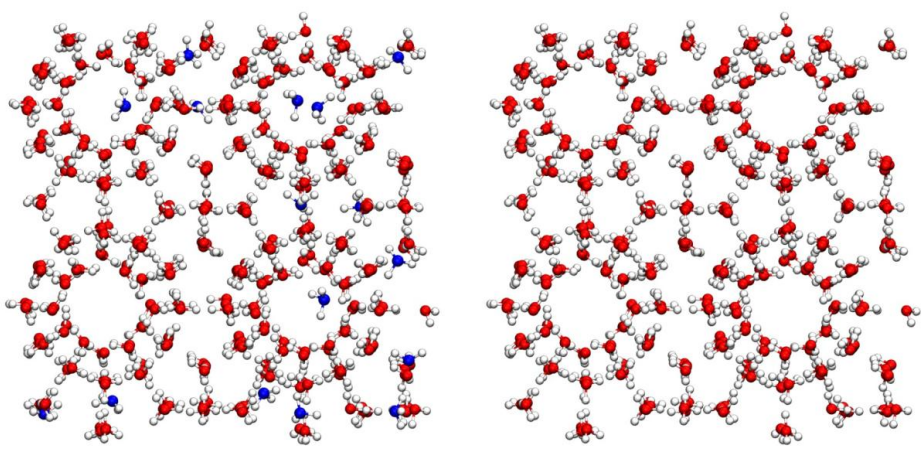

(b)
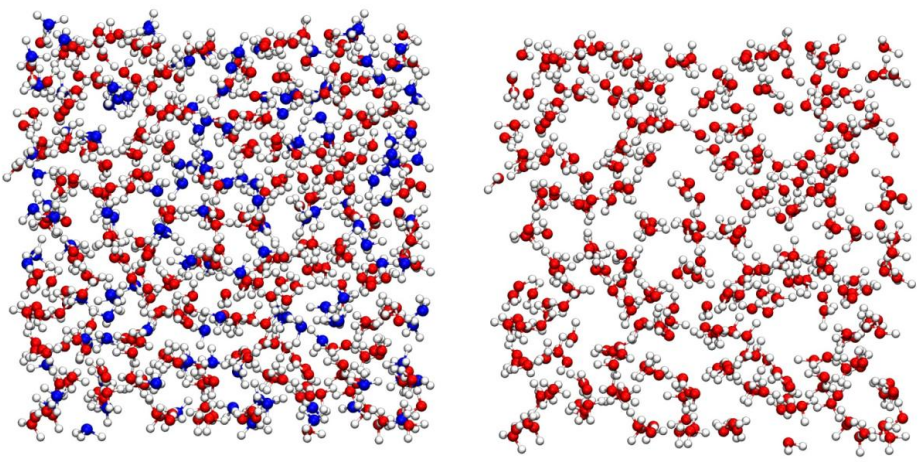

Fig. 2. Some snapshots of the systems in a flexible clathrate hydrate of $\mathrm{NH}_{3}$, as obtained from our GCMC simulations at $100 \mathrm{~K}$ at (a) low $(-21.72 \mathrm{~kJ} / \mathrm{mol})$ and (b) high $(-10.08 \mathrm{~kJ} / \mathrm{mol})$ chemical potential values corresponding to different fillings of the cages. $\mathrm{O}, \mathrm{N}$, and $\mathrm{H}$ atoms are shown by red, blue and white balls, respectively. Note that snapshots for the same chemical potential values are given on the right hand side of the figure, in which ammonia molecules have been removed for clarity (i.e., only the water network is shown)

\subsection{Structural Characterization}

To investigate the structure of the ammonia+water system when the ammonia content increases, we have characterized the geometrical order of the water molecules in the simulation box by calculating an order parameter that measures the degree to which the nearest-neighbor water molecules are tetrahedrally coordinating a reference water molecule [42]. The tetrahedral 
order parameter that has been shown to correctly describe the ordering of the water molecules in clathrates [42] has been defined as follows:

$$
<\zeta^{w w}>=\frac{1}{N_{w}} \sum_{N_{w}}\left[1-\frac{3}{8} \sum_{i=1}^{3} \sum_{j=i+1}^{4}\left(\cos \psi_{i j}+1 / 3\right)^{2}\right]
$$

where the indices $i$ and $j$ run over the four nearest water neighbours of a given water molecule, $\Psi_{i j}$ is the angle between the bonds that this given molecule forms with its nearest-neighbors $i$ and $j$, and $N_{W}$ is the total number of water molecules. For a perfectly ordered clathrate, $\zeta^{\mathrm{ww}}=1$, whereas this value decreases to 0.63 in liquid water.[42]

The values of $\left\langle\zeta^{\mathrm{ww}}\right\rangle$ calculated at 100,150 and $180 \mathrm{~K}$ in the range of chemical potential considered are given in Figure 3, together with the occupancy isotherms for the flexible clathrates.

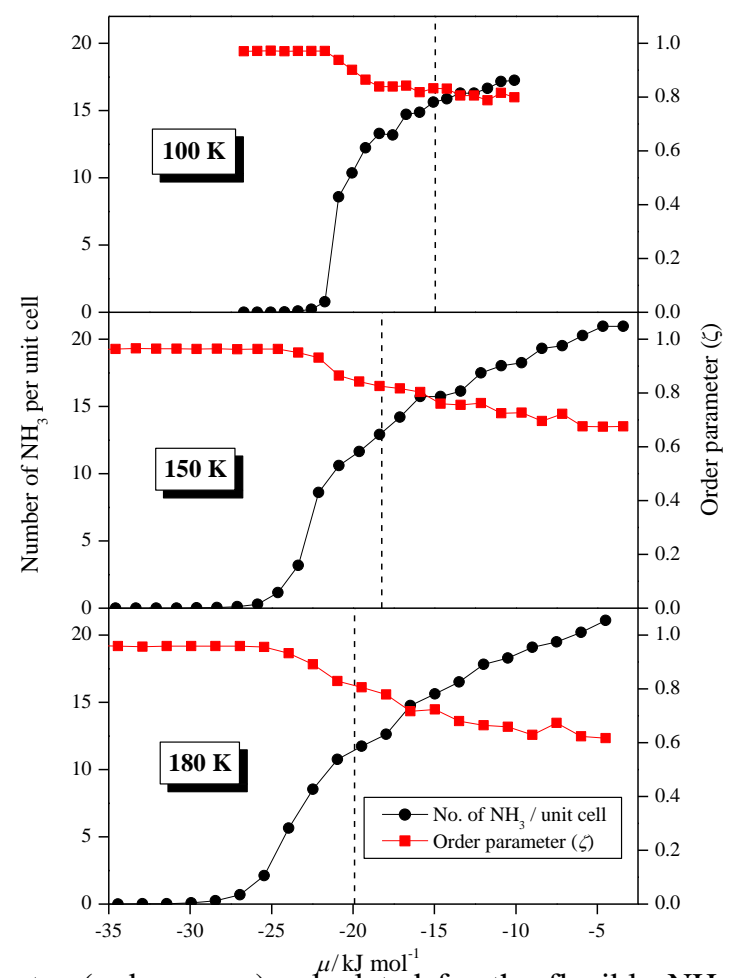

Fig. 3. Tetrahedral order parameter (red squares) calculated for the flexible $\mathrm{NH}_{3}$ clathrates, as a function of the chemical potential, at $100 \mathrm{~K}$ (top panel), $150 \mathrm{~K}$ (middle panel), and $180 \mathrm{~K}$ (bottom panel). The corresponding occupancy isotherms are also shown (black circles). The lines connecting the points are just guides to the eye. The dashed vertical lines indicate the chemical potential values corresponding to the point of condensation of $\mathrm{NH}_{3}$. $\mathrm{Scales}$ at the left and right side of the figure correspond to the occupancy isotherm and order parameter data, respectively. Note that, for the ordinate axis, we divide the number of ammonia molecules in the simulation box by the number of unit cells of the rigid clathrate, as an arbitrary normalization. 
These curves clearly indicate that the values of $\left\langle\zeta^{\mathrm{ww}}\right\rangle$ are equal to or slightly smaller than one up to a threshold value of the chemical potential, above which they continuously decrease. More interestingly, this threshold value, which is obtained at the beginning of the rising part of the isotherm corresponds to around 8 ammonia molecules per unit cell at 100 and $150 \mathrm{~K}$, and to a number of $\mathrm{NH}_{3}$ between about 5 and 8 molecules per unit cell at $180 \mathrm{~K}$. This corresponds approximately to a complete filling of the clathrate structure (i.e., one molecule per cage in average) at the two lowest temperatures considered here, and, more probably, to a partial filling of the clathrate at $180 \mathrm{~K}$. Then, above this threshold value, the decrease of $\left\langle\zeta^{\mathrm{ww}}\right\rangle$ could be interpreted as resulting from an increasing disordering of the water network when the number of enclathrated $\mathrm{NH}_{3}$ molecules increases. However, the value $\left\langle\zeta^{\mathrm{wW}}\right\rangle=0.6$, corresponding to a complete breakdown of the clathrate structure, such as in liquid water, is only reached at $180 \mathrm{~K}$ and very high $\mathrm{NH}_{3}$ loading, which rises questions on the physical state of the systems corresponding to intermediate values of $\left\langle\zeta^{\mathrm{ww}}\right\rangle$ between 1 (perfectly tetrahedrally ordered water molecules) and 0.6 (liquid-like disordered water molecules).

A more detailed analysis of the water arrangements can, in fact, be deduced from the distribution of the $\zeta^{\mathrm{ww}}$ parameter values, $P\left(\zeta^{\mathrm{ww}}\right)$, than simply from its average value. Let us first comment the situation corresponding to the results obtained at $100 \mathrm{~K}$. In this case, the $P\left(\zeta^{\mathrm{ww}}\right)$ distribution is given in Figure 4 for selected values of the chemical potential, $(\mu=-21.72,-20.88$, $-19.22,-16.73,-13.40$, and $-10.08 \mathrm{~kJ} / \mathrm{mol})$ corresponding to different $\mathrm{NH}_{3}$ loadings $(0.8,8.6$, 12.2, 14.7, 16.3 and 17.2 $\mathrm{NH}_{3}$ molecules per unit cell in average, respectively). At low chemical potential values, the calculated distribution clearly exhibits one single peak, located at 0.997 , indicating that in this case all the water molecules are involved in a tetrahedral arrangement with their water neighbors. In contrast, when increasing the chemical potential value (i.e., when increasing the number of $\mathrm{NH}_{3}$ molecules trapped in the system), a small shift of $\zeta^{\mathrm{ww}}$ to lower values is observed and, more importantly, a second peak is evidenced at around $\zeta^{\mathrm{wW}}=0.4$ in the distribution, the intensity of which is increasing with the ammonia loading. The presence of this second peak indicates that an increasing fraction of water molecules lose their tetrahedral coordination when the number of ammonia molecules increases. However, the integration of this peak of the $P\left(\zeta^{\mathrm{ww}}\right)$ distribution shows that about $80 \%$ of the water molecules remain more or less involved in a tetrahedral arrangement with the surrounding water molecules even at the highest chemical potential values considered here (corresponding to much more than one $\mathrm{NH}_{3}$ molecules 
per clathrate cage, as indicated by the isotherm given in Figure 3 (top)). This conclusion clearly indicates that the average value of $\left\langle\zeta^{\mathrm{ww}}\right\rangle=0.8$, calculated at $100 \mathrm{~K}$ at high chemical potentials (Figure 3 (top)) does not correspond to a fully disordered system, but rather to a tetrahedrally ordered system, containing non-tetrahedrally coordinated, possibly off-network water molecules up to $20 \%$. This conclusion suggests the persistence of the clathrate structure in the simulation box during the entire course of our simulations, even at very high ammonia loading, as evidenced on the simulation snapshots (Figure 2).

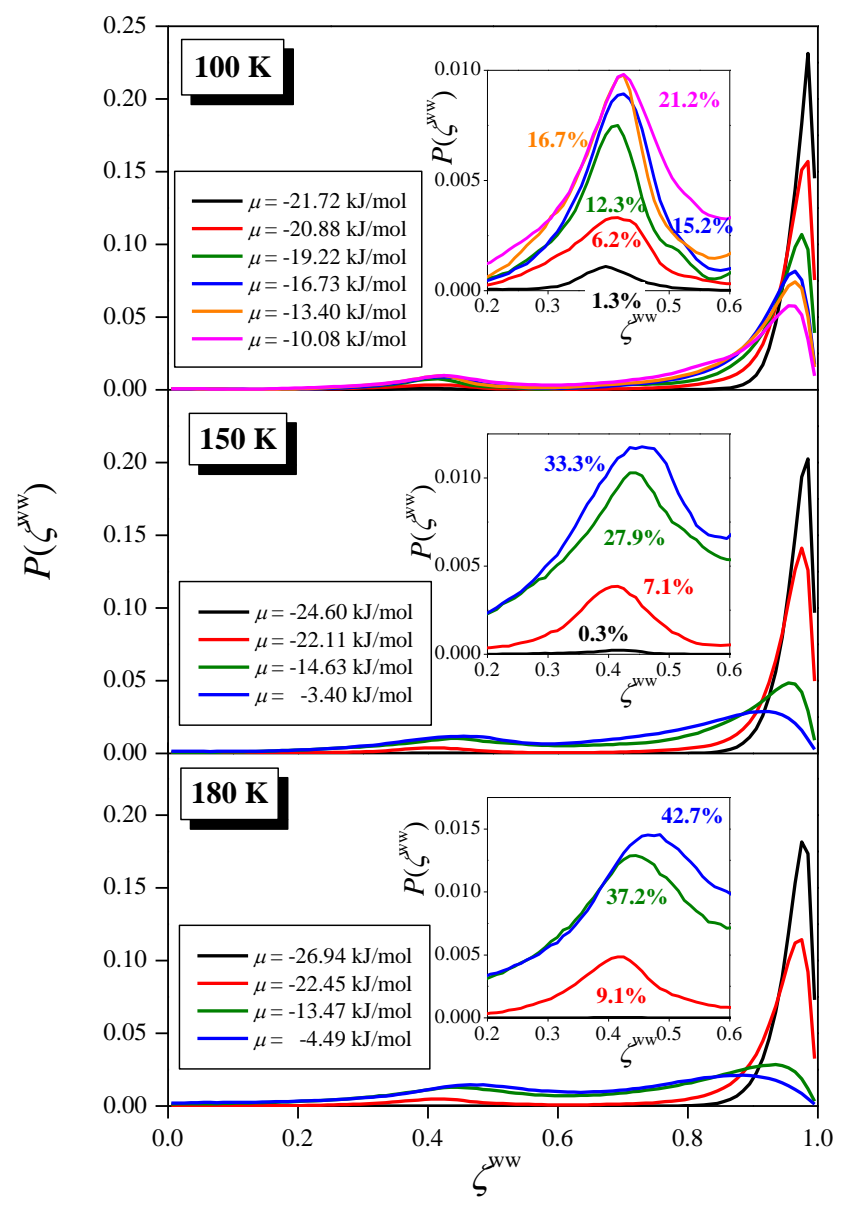

Fig. 4. Distributions of the tetrahedral order parameter, obtained by considering only the $\mathrm{O}$ atoms, for various chemical potential values, corresponding to different $\mathrm{NH}_{3}$ loadings, in the flexible clathrate at $100 \mathrm{~K}$ (top panel), $150 \mathrm{~K}$ (middle panel), and $180 \mathrm{~K}$ (bottom panel). The insets show the region of the peak around 0.4 on a magnified scale. The percentage of atoms giving rise to this peak is also indicated.

To complement our investigations, we have also calculated the tetrahedral order parameter without discriminating between water or ammonia neighbors of the reference water 
molecule. The corresponding distributions of this parameter are given in Figure 5 (top) at different chemical potential values. To distinguish it from the $\zeta^{\mathrm{ww}}$ order parameter, measuring the tetrahedrality of solely the water neighbors, this parameter is denoted here as $\zeta^{\mathrm{N}}$. Contrarily to $P\left(\zeta^{\mathrm{ww}}\right)$, the $P\left(\zeta^{\mathrm{w}}\right)$ distributions are characterized by only a single peak, the maximum of which is always located above 0.9. Although this peak exhibits an increasing shoulder at lower values when the chemical potential value rises, the persistence of the main peak around 0.95 shows that most of the water molecules stay tetrahedrally coordinated by their neighbors in the entire chemical potential range investigated. This is a strong indication that some ammonia molecules can replace water molecules, and become incorporated in the water lattice, as already observed in previous molecular dynamics simulations of the ammonia clathrate.[11]

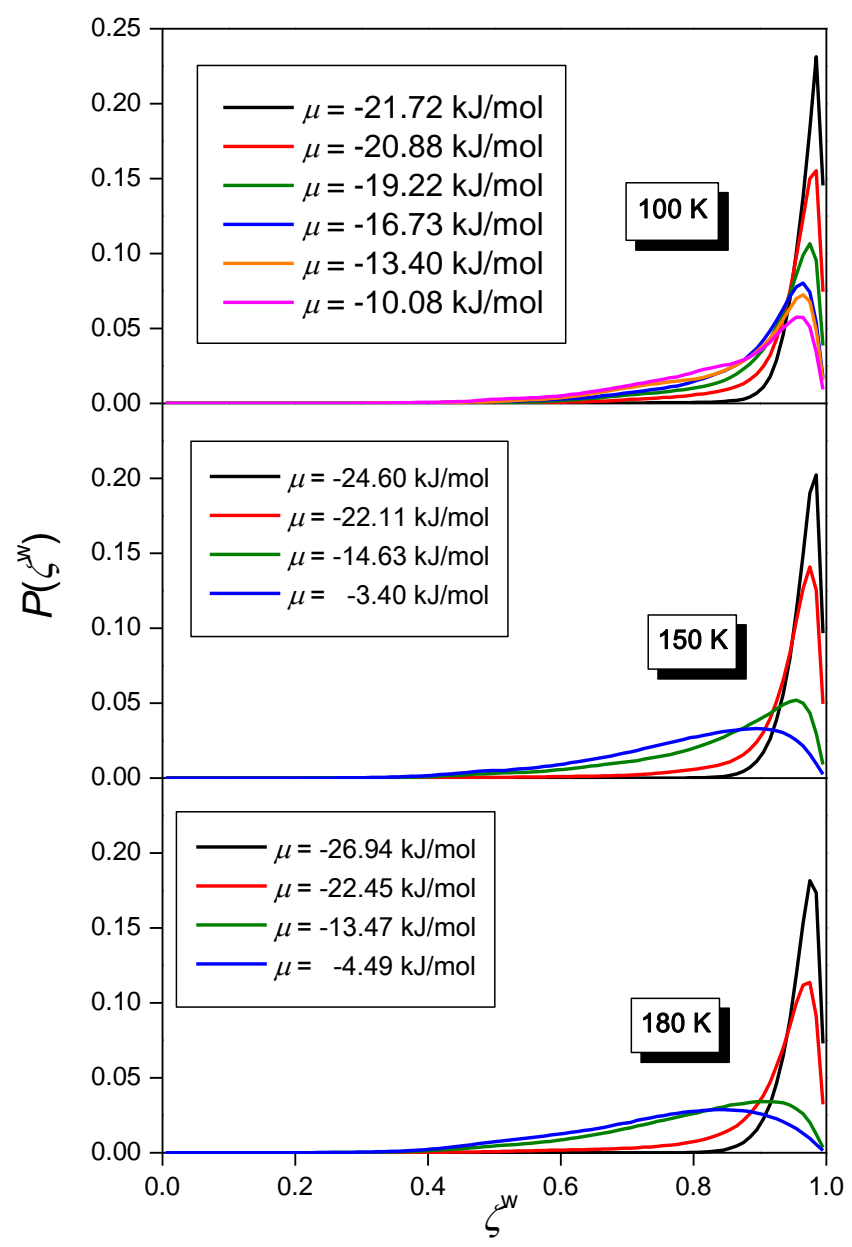

Fig. 5. Distribution of the tetrahedral order parameter, obtained by considering both the $\mathrm{O}$ and $\mathrm{N}$ atoms, for various chemical potential values, corresponding to different $\mathrm{NH}_{3}$ loadings, in the flexible clathrate at $100 \mathrm{~K}$ (top panel), $150 \mathrm{~K}$ (middle panel) and $180 \mathrm{~K}$ (bottom panel). 
Because the tetrahedral coordination cannot unambiguously discriminate between ice-like and clathrate-like configurations, an additional criterion characterizing the obtained structure is needed. Such a criterion is based on the fact that water molecules in clathrates form fivemembered rings (cyclic pentamers) in clathrates, while no such cyclic pentamers exist in $I_{h}$ ice.[43] A water molecule is thus considered being involved in a cyclic pentamer if a structure is found in which (i) five oxygen atoms of the water molecules form the vertices of such a ring, and (ii) the length of all the five edges of this ring structure is shorter than the position of the first minimum in the oxygen-oxygen radial distribution function in the system.[43] According to this criterion, water molecules in $I_{h}$ ice do not form any five-member rings, while in clathrate structures all water molecules participate in 4,5 , or 6 such rings. Note also that liquid water shows a broad distribution of cyclic pentamers.[43]

The calculated fraction of water molecules that are part of a given number of pentameric rings at $100 \mathrm{~K}$ is shown in Figure 6a for selected values of the chemical potential, corresponding to different $\mathrm{NH}_{3}$ loadings along the occupancy isotherm.
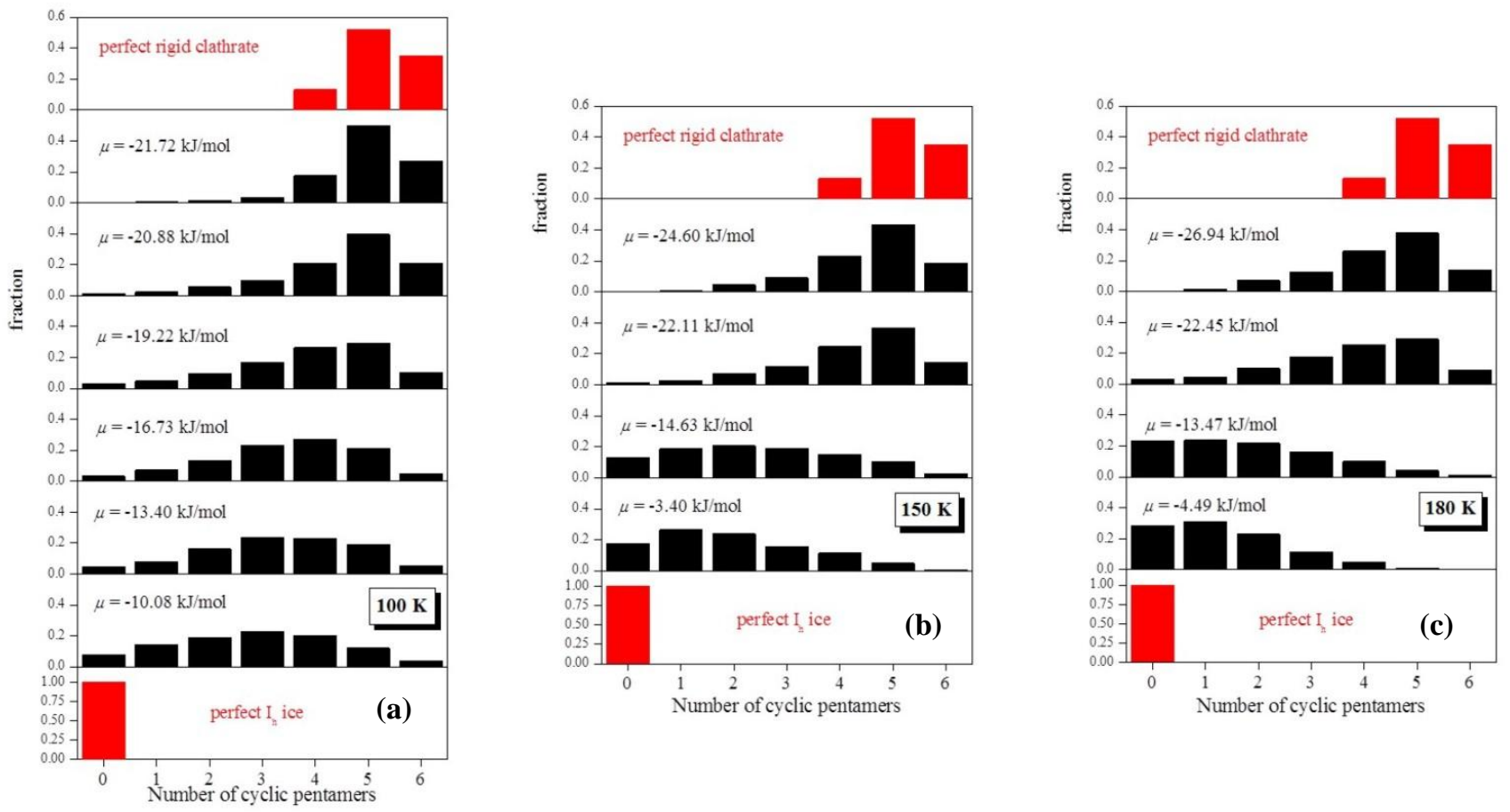

Fig. 6. Fraction of water molecules that belong to a given number of cyclic pentamers in the systems simulated at (a) $100 \mathrm{~K}$, (b) $150 \mathrm{~K}$ and (c) $180 \mathrm{~K}$ at various chemical potential values, corresponding to different $\mathrm{NH}_{3}$ loadings. The distributions corresponding to the perfect rigid clathrate and to the perfect $\mathrm{I}_{\mathrm{h}}$ ice crystal are given as a reference in the top and bottom panels, respectively. 
The reference result for a rigid clathrate is also shown in the figure (top of Figure 6a), which confirms that water molecules in a perfectly ordered clathrate structure are involved in 4, 5 or 6 pentamers. In contrast, when considering flexible clathrates, an increasing fraction of water molecules belonging to less than 4 cyclic pentamers is calculated with increasing ammonia chemical potential (and, hence, with increasing ammonia loading), which is a strong indication for a progressive loss of connections between adjacent cages. Nevertheless, even at the highest ammonia loading, a significant number of water molecules still take part in 4, 5 or 6 pentameric rings, indicating that the clathrate structure is partially preserved in our system.

Another way to characterize the structural arrangement of the molecules in the systems investigated is the calculation of certain partial radial distribution functions (RDFs). Figure 7a shows the simulated RDFs between the oxygen atoms of the water cages $\left(g_{\mathrm{OO}}(r)\right)$, between these oxygen atoms and the nitrogen atoms of the ammonia molecules $\left(g_{\mathrm{NO}}(r)\right)$, and between nitrogen atoms of the ammonia molecules $\left(g_{\mathrm{NN}}(r)\right)$ for different values of the chemical potential, corresponding to various ammonia loadings in the flexible clathrate at $100 \mathrm{~K}$. For comparison, results obtained at full loading (i.e., when each cage is occupied by one $\mathrm{NH}_{3}$ molecule) of the rigid clathrate is also shown. First, $g_{\mathrm{OO}}(r)$ (top of Figure 7a) shows that, at the low chemical potential values corresponding to the first part of the occupancy isotherm, the structural arrangement of the water molecules is similar in the rigid and flexible clathrates. This is a clear indication of the preservation of the clathrate structure at low ammonia loading. However, as the chemical potential value increases, i.e., more and more $\mathrm{NH}_{3}$ molecules are present in the simulated system, the long-range ordering of the oxygen atoms gets progressively lost. Meanwhile, the first peak of the N-N RDFs shows that nearest neighbor ammonia molecules are separated by about $6.5 \AA$ at low $\mathrm{NH}_{3}$ loading, corresponding to the very first part of the occupancy isotherm (i.e., at low chemical potential values), similarly to that in the rigid clathrate. This distance corresponds to that of the neighboring cages. However, as the number of the trapped $\mathrm{NH}_{3}$ molecules increases, an additional peak of $g_{\mathrm{NN}}(r)$ is evidenced around $3 \AA$ (bottom of Figure 6a), indicating that $\mathrm{NH}_{3}$ pairs might have been formed in the system. This feature could correspond to near-neighbor ammonia molecules in a more and more disordered structure. Finally, the analysis of the $g_{\mathrm{NO}}(r)$ functions also confirms that the flexible clathrate behaves similarly to the rigid one at low chemical potential values, whereas significant differences are evidenced when the number of the ammonia molecules increases (middle of 
Figure 6a). In particular, the first peak of $g_{\mathrm{NO}}(r)$ around $4 \AA$ not only broadens and shifts to smaller distances (i.e. down to $3.4 \AA$ ) when the chemical potential value increases, but it also exhibits a weak shoulder around $2.8 \AA$. These features are clear indications of the formation of some $\mathrm{NH}_{3}-\mathrm{H}_{2} \mathrm{O}$ pairs in the system, and can be related to the conclusions obtained above from the calculations of the $\zeta^{\mathrm{w}}$ parameter, namely that some of the ammonia molecules can displace water, and become incorporated in the water lattice, contributing to the increase of the number of ammonia molecules trapped in the simulated system with increasing the chemical potential values.

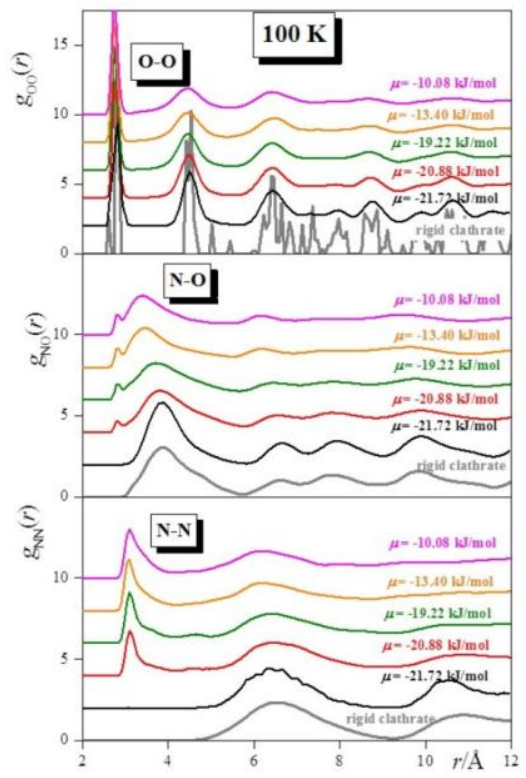

(a)

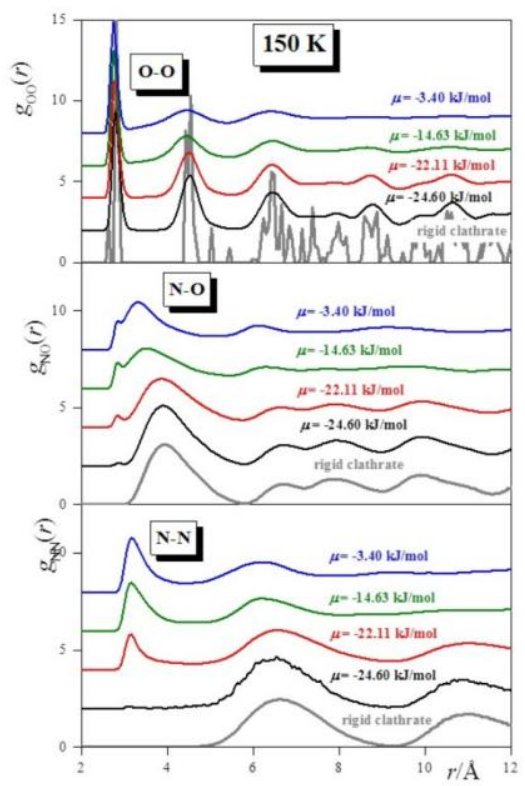

(b)

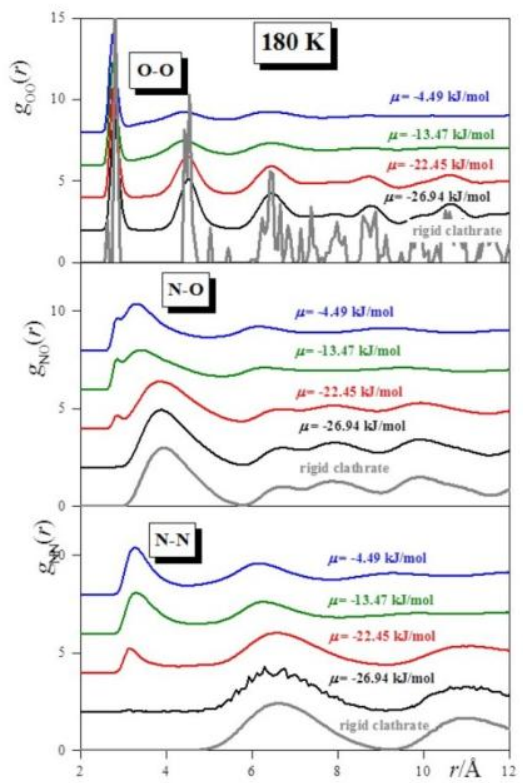

(c)

Fig. 7. Partial radial distribution functions between the $\mathrm{O}$ atoms of the water cages (top panels), between the $\mathrm{O}$ and $\mathrm{N}$ atoms (middle panels), and between the $\mathrm{N}$ atoms (bottom panels), as obtained at (a) $100 \mathrm{~K}$, (b) $150 \mathrm{~K}$ and (c) $180 \mathrm{~K}$ at various chemical potential values, corresponding to different $\mathrm{NH}_{3}$ loadings. The radial distribution functions calculated for the rigid clathrate at full occupancy is also given as a reference. The curves corresponding to increasing chemical values are shifted up by $2,4,6,8$, and 10 units, for clarity.

To get more information on this $\mathrm{NH}_{3}-\mathrm{H}_{2} \mathrm{O}$ bonding, we have thus calculated the pair energy distributions for the molecules that are closer than $3.5 \AA$. The corresponding curves (not shown) exhibit two peaks, one at around $-29 \mathrm{~kJ} / \mathrm{mol}$ and another one at about $-10 \mathrm{~kJ} / \mathrm{mol}$, indicating two different modes of bonding between $\mathrm{NH}_{3}$ and water molecules at short distances. 
By comparison with the literature data for the $\mathrm{NH}_{3}-\mathrm{H}_{2} \mathrm{O}$ dimer,[44-46] the former value (- 29 $\mathrm{kJ} / \mathrm{mol}$ ) can be attributed to the formation of $\mathrm{NH}_{3}-\mathrm{H}_{2} \mathrm{O}$ dimers in which the water molecule acts as a H-bond donor (corresponding to the O-N distance of about $2.9 \AA$ ), whereas the later value ($10 \mathrm{~kJ} / \mathrm{mol}$ ) can be related to such H-bonded dimers in which the water molecule acts as a proton acceptor (the corresponding $\mathrm{O}-\mathrm{N}$ distance is about $3.2 \AA$ ).

Similar analyses have been performed at the two higher temperatures considered, i.e., 150 and $180 \mathrm{~K}$, to characterize the structural evolution of the ammonia clathrate upon temperature increase. According to the shape of the occupancy isotherm simulated at $T=150 \mathrm{~K}$, four characteristic systems have been analyzed in detail. These systems correspond to the chemical potential values of $\mu=-24.6,-22.11,-14.63$, and $-3.40 \mathrm{~kJ} / \mathrm{mol}$, and to $1.1,8.6,15.7$ and $20.6 \mathrm{NH}_{3}$ molecules per unit cell, respectively. The corresponding distributions of the tetrahedral order parameter for the relative arrangement of the neighboring water molecules, $P\left(\zeta^{\mathrm{ww}}\right)$, the distribution of the tetrahedral order parameter without discriminating between water or ammonia neighbors of the reference water molecule, $P\left(\zeta^{\mathrm{w}}\right)$, and the fraction of water molecules that form part of a given number of pentameric rings are shown in Figure 4 (middle panel), Figure 5 (middle panel) and Figure 6b, respectively. As is evidenced in these figures, the clathrate structure appears to be almost perfectly preserved up to a loading corresponding to about 8 ammonia molecules per unit cell, with a loss of the tetrahedral coordination for about $7 \%$ of the water molecules, corresponding to an increasing fraction of water molecules that are involved in less than 4 cyclic pentamers. The higher ammonia loadings are characterized by an increasing disorder, and the complete disappearance of water molecules involved in 6 pentameric rings is a strong indication for the disruption of the clathrate structure at $150 \mathrm{~K}$. This is also confirmed by the disappearance of the peak around 0.9 in $P\left(\zeta^{\mathrm{w}}\right)$, which illustrates the loss of any tetrahedral coordination for the water molecules as the number of ammonia molecules increases in the simulation box. Meanwhile, the increasing number of water molecules that are not included in any cyclic pentamer (as evidenced by the growing intensity of the peak at 0 in Figure 7) suggests the formation of ice in the system as the clathrate structure disappears. These conclusions are also supported by the analysis of the $g(r)$ curves given on Figure 7b. Indeed, the $g_{\mathrm{OO}}(r)$ functions show that while the clathrate structure is almost preserved at $\mu=-24.6$ and $-22.11 \mathrm{~kJ} / \mathrm{mol}$ (i.e., $<\mathrm{N}\rangle=1.1$ and 8.6 ammonia molecules per unit cell, respectively), it clearly disappears at higher chemical potential values, at which $g_{\mathrm{OO}}(r)$ appears to be similar to the corresponding distribution 
calculated for low density amorphous (LDA) ice.[47,48] In addition, the appearance of a short

distance peak of $g_{\mathrm{NN}}(r)$ at $3.2 \AA$ as well as of that of $g_{\mathrm{NO}}(r)$ around $2.8 \AA$ indicate the formation of ammonia-ammonia and ammonia-water pairs in the system.

Similar trends are clearly evidenced at $180 \mathrm{~K}$ (see Figure 4 (bottom panel), Figure 5 (bottom panel) Figures 6c and 7c, where the results are given for the $\mu$ values of $-26.94,-22.45$, -13.47, and $-4.49 \mathrm{~kJ} / \mathrm{mol}$, corresponding to an average of $0.7,8.5,16.5$, and $21.1 \mathrm{NH}_{3} \mathrm{molecules}$ per unit cell, respectively). Indeed, even at this high temperature, a noticeable part of the system still exhibits clathrate structure features up to the loading of about $8 \mathrm{NH}_{3}$ molecules per unit cell (at least during the entire course of our simulations). By contrast, larger loadings are characterized by the disappearance of the clathrate structure, the concomitant formation of an ice phase exhibiting $g_{\mathrm{OO}}(r)$ similar to LDA ice, and the increase of the number of ammonia-ammonia and ammonia-water pairs in the system.

\section{Summary and Conclusions}

In this paper, we have investigated the trapping of ammonia molecules into a clathrate structure by means of Grand Canonical Monte Carlo simulations, performed at three different temperatures relevant for astrophysical environments. Contrarily to previous molecular dynamics simulation studies performed on the same system,[10,11] no a priori assumption was made on the number of ammonia molecules incorporated into the water lattice, which was, by contrast, calculated directly from the simulations as a function of the chemical potential. This treatment allowed us to investigate the stability of the clathrate structure as a function of not only the temperature, but also of the ammonia loading.

Our results have revealed that ammonia containing clathrate of structure $I$ is stable at partial filling during the whole length of our GCMC simulations, irrespective of the temperature in the range investigated here. It could also be stable (or, more exactly, metastable during the entire course of our simulations) in a chemical potential range (and hence in a pressure range) that corresponds to a maximum of 8 ammonia molecules per unit cell, i.e., to the full occupancy of the clathrate structure. At higher chemical potential values and higher temperatures, new features are evidenced by the various structural analyses performed, which can be related to a progressive dissolution of the clathrate concomitant with a transformation to an ice phase 
showing structural ordering similar to low density amorphous ice at 150 and $180 \mathrm{~K}$. This dissolution of the clathrate allows a continuously increasing loading of ammonia molecules that are thus mixed with water molecules in the new phase formed. It should be mentioned that the full characterization of the ice phase form upon clathrate dissolution would require additional investigations that are beyond the scope of this paper, since discriminating between the various phases of solid ice could be a very tedious task.[49] In addition, the present results may depend on the water potential model used here (TIP4P/Ice), for which no detailed characterization of the various amorphous ice phases is available in the literature, contrarily to a number of water models, including polarizable ones, [50] which have been shown to exhibit stability of LDA ice up to at least $180 \mathrm{~K}$, depending also on the pressure and the way of preparation.[51-54] Moreover, as for any GCMC simulations, the results obtained could depend on the number of MC steps used in the calculations. Although, very long simulations, involving a huge number of MC steps have been performed here, it could be possible that the total decomposition of the clathrate hydrate phase upon increasing ammonia loading has not been fully reached during the course of our simulations. In that sense; the picture described here in which part of the system remains clathrate-like at 150 and $180 \mathrm{~K}$ even at high ammonia loading could be questioned in much longer GCMC simulations.

Anyway, the present GCMC results support the stability (or metastability) of the ammonia clathrate of structure I at very low temperature $(100 \mathrm{~K})$ and for chemical potential (i.e., pressure) values that do not exceed the single-occupancy of all the clathrate cages, in accordance with the conclusions obtained from previous MD simulations.[10,11].

However, that we cannot definitely rule out the possibility that our system has been stuck in a local free energy minimum during the entire course of our simulations (as it could also be the case in MD simulations). Note that such metastable states have been recently experimentally observed, for instance for the $\mathrm{N}_{2}$ clathrate,[55] and, even, for empty clathrates,[56] suggesting that kinetic effects may play an important role in the evolution of the clathrates structures.[55]

In the systems simulated in the present work, ammonia molecules are trapped into the cages of the water lattice with the formation of hydrogen-bonds between water and ammonia molecules, in which the water molecule can act as both proton-acceptor and proton-donor. Ammonia molecules can also displace water molecules, and become incorporated into the water lattice upon increasing the ammonia loading and the temperature. The incorporation of some 
ammonia molecules into the water lattice results in the destabilization of the clathrate structure and, as the number of ammonia molecules increases, more and more ammonia-ammonia and ammonia-water pairs are formed that finally lead to the disappearance of the clathrate structure.

The results of the present work emphasize the role that ammonia can play in forming or destabilizing solid clathrate phases at low temperatures in planetary environments.[57] They could thus help in better understanding the mechanisms governing the stability regions of, for instance, the methane clathrate phases on various moons in the Solar System.[10] Indeed,

methane clathrates are likely present on, e.g., Titan, Enceladus, or Europa,[58] where they are supposed to consist internal reservoirs of methane and other atmospheric gases. In the case of Titan, the dissociation of these methane clathrates has even been invoked to be the main contributor to the replenishment of Titan's atmospheric methane via outgassing episodes.[58-60].

Our calculations also point out the subtle interplay between the various environmental conditions (temperature, partial pressure of ammonia) on the stability of the ammonia clathrate phase. Ammonia clathrate may thus be ubiquitous in the interiors of the icy bodies of the outer solar system. Indeed, the surface temperature of Jupiter's icy moon Europa, which can reach about $130 \mathrm{~K}$, [61] suggests that ammonia clathrate may be present in the ice crust and then favor some type of biochemistry. The subsurfaces of other icy bodies of the solar system located at further distances may also harbor ammonia clathrates, provided that some internal heat sources allow the local temperature to remain in the 100-150 K range.

\title{
Supporting Information
}

Data of the occupancy isotherm of ammonia in clathrate hydrate at $100 \mathrm{~K}$ (Table S1), $150 \mathrm{~K}$ (Table S2), and $180 \mathrm{~K}$ (Table S3) for the system corresponding to $2 \times 2 \times 2$ clathrate units in the basic box, and at $100 \mathrm{~K}$ (Table S4), $150 \mathrm{~K}$ (Table S5) and $180 \mathrm{~K}$ (Table S6) for the system corresponding to $3 \times 3 \times 3$ clathrate units in the basic box. (PDF)

\author{
Author Information \\ Corresponding Author \\ *E-mail: sylvain.picaud@univ-fcomte.fr
}


Acknowledgements. This project is supported by the CNRS in the framework of an international program for scientific cooperation (PICS), by the Hungarian NKFIH Foundation under project No. 119732, by the Hungarian-French Intergovernmental Science and Technology Program (BALATON) under project No. TéT_15_FR-1-2016-0056, and by the French National Research Agency (ANR) in the framework of the MI2C project. This work has been partly carried out thanks to the support of the A*MIDEX project ( $n^{\circ}$ ANR-11-IDEX-0001-02) funded by the "Investissements d'Avenir" French Government program, managed by the French National Research Agency (ANR). Calculations have been performed on computers from the UTINAM Institute supported by the Region de Franche-Comte and Institut des Sciences de l'Univers (INSU) - CNRS and also from the Mésocentre de Calcul, a Regional Computing Center at Université de Franche-Comté.B. F. is supported by the ÚNKP-17-3-I New National Excellence Program of the Ministry of Human Capacities of Hungary. O.M. acknowledges support from CNES. 


\section{References}

(1) Ho, P.T.P; Townes, C.H. Interstellar Ammonia. Ann. Rev. Astron. Astrophys. 1983, 21, 239-270.

(2) Lewis, J.S. Physics and Chemistry of the Solar System. 1995, Academic Press, Inc. London.

(3) Atreya, S.K.; Donahue, T.M.; Kuhn, W.R. Evolution of a Nitrogen Atmosphere on Titan. Science 1978, 201, 611-613.

(4) Meringer, M.; Cleaves, H.J.; Freeland, S.J. Beyond Terrestrial Biology: Charting the Chemical Universe of $\alpha$-Amino Acid Structures. J. Chem. Inf. Model 2013, 53, 28512862.

(5) Lunine, J.I.; Stevenson, D.J. Clathrate and Ammonia Hydrates at High Pressure: Application to the Origin of Methane on Titan. Icarus 1987, 70, 61-77.

(6) Grasset, O.; Pargamin, J. The ammonia-water system at high pressures: Implications for the methane of Titan. Planet. Space Sci. 2005, 53, 371-384

(7) Cook, J.C.; Desch, S.J.; Roush, T.L.; Trujillo C.A.; Geballe, T.R. Near-Infrared Spectroscopy of Charon : Possible Evidence for Cryovolcanism on Kuiper Belt Objects. ApJ 2007, 663, 1406-1419.

(8) Delsanti, A.; Merlin, F.; Guilbert-Lepoutre, A.; Bauer, J.; Yang, B.; Meech, K.J. Methane, Ammonia, and Their Irradiation Products at the Surface of an Intermediate-Size KBO ? A Portrait of Plutino (90482) Orcus. A\&A 2010, 520, A40.

(9) Jewitt, D.C.; Luu, J. Crystalline Water Ice on the Kuiper Belt Object (50000) Quaoar. Nature 2004, 432, 731-733.

(10) Shin, K.; Kumar, R.; Udachin, K.A.; Alavi, S.; Ripmeester, J.A. Ammonia Clathrate Hydrates as New Solid Phases for Titan, Enceladus, and Other Planetary Systems. Proc. Natl. Acad. Sci. USA 2012, 109, 14785-14790.

(11) Alavi, S.; Shin, K.; Ripmeester, J.A. Molecular Dynamics Simulations of Hydrogen Bonding in Clathrate Hydrates with Ammonia and Methanol Guest Molecules. J. Chem. Eng. Data 2015, 60, 389-397.

(12) Adams, D. J. Grand Canonical Ensemble Monte Carlo for a Lennard-Jones Fluid. Mol. Phys. 1975, 29, 307-311.

(13) Allen, M. P.; Tildesley, D. J. Computer Simulation of Liquids; Clarendon: Oxford, 1987.

(14) Tanaka, H. The Thermodynamics Stability of Clathrate Hydrate. III. Accommodation of Nonspherical Propane and Ethane Molecules. J. Chem. Phys. 1994, 101, 10833

(15) Tanaka, H. A Novel Approach to the Stability of Clathrate Hydrates: Grand Canonical Monte Carlo Simulation. Fluid Phase Equilibria 1998, 144, 361-368.

(16) Sizov, V.V.; Piotrovskaya, E.M. Computer Simulation of Methane Hydrate Cage Occupancy. J. Phys. Chem. B 2007, 111, 2886-2890. 
(17) Wierzchowski, S.J.; Monson, P.A. Calculation of Free Energies and Chemical Potentials for Gas Hydrates Using Monte Carlo Simulations. J. Phys. Chem. B 2007, 111, 72747282.

(18) Katsumasa, K.; Koga, K.; Tanaka, H. On the Thermodynamic Stability of Hydrogen Clathrate Hydrates. J. Chem. Phys. 2007, 127, 044509.

(19) Papadimitriou, N. I.; Tsimpanogiannis, I. N.; Papaioannou, A. Th.; Stubos, A. K. Evaluation of the Hydrogen-Storage Capacity of Pure $\mathrm{H}_{2}$ and Binary $\mathrm{H}_{2}$-THF Hydrates with Monte Carlo Simulations. J. Phys. Chem. C 2008, 112, 10294-10302.

(20) Lectez, S.; Simon, J.M.; Mousis, O.; Picaud, S.; Altwegg, K.; Rubin, M.; Salazar, M. A 32-70 K Formation Temperature Range for the Ice Grains Agglomerated by Comet 67P/Churyumov-Gerasimenko. Astrophys. J. Lett. 2015, 805, L1.

(21) Takeuchi, F.; Hiratsuka, M.; Ohmura, R.; Alavi, S.; Sum, A.K.; Yasuoka, K. Water Proton Configurations in Structures I, II, and H Clathrate Hydrate Unit Cells. J. Chem. Phys. 2013, 138, 124504.

(22) Abascal, J.L.F.; Sanz, E; García Fernández, R.; Vega, C. A Potential Model For the Study of Ices and Amorphous Water: TIP4P/Ice. J. Chem. Phys. 2005, 122, 234511.

(23) Conde, M.M.; Vega; C. Determining the three-phase coexistence line in .methane hydrates using computer simulations. J. Chem. Phys. 2010, 133, 064507.

(24) Rizzo, R.C.; Jorgensen, W.L. OPLS All-Atom Model for Amines: Resolution of the Amine Hydration Problem. J. Am. Chem. Soc. 1999, 121, 4827 - 4836.

(25) Barker, JA; Watts, RO. Monte Carlo Studies of the Dielectric Properties of Water-Like Models. Mol. Phys. 1973, 26, 789 - 792

(26) Neumann, M. The Dielectric Constant of Water. Computer Simulations with the MCY Potential. J. Chem. Phys. 1985, 82, 5663 - 5672.

(27) M. Mezei, M. MMC: Monte Carlo Program for Simulation of Molecular Assemblies. URL: http://inka.mssm.edu/ mezei/mmc.

(28) Jedlovszky, P.; Partay, L.; Hoang, P. N. M.; Picaud, S.; von Hessberg, P.; Crowley, J. N. Determination of the Adsorption Isotherm of Methanol on the Surface of Ice. An Experimental and Grand Canonical Monte Carlo Simulation Study. J. Am. Chem. Soc. 2006, 128, 15300-15309.

(29) Jedlovszky, P.; Hantal, Gy.; Neuróhr, K.; Picaud, S.; Hoang, P. N. M.; von Hessberg, P.; Crowley, J. N. Adsorption Isotherm of Formic Acid on The Surface of Ice, as Seen from Experiments and Grand Canonical Monte Carlo Simulation. J. Phys. Chem. C 2008, 112, 8976-8987.

(30) Darvas, M.; Lasne, J.; Laffon, C.; Parent, P.; Picaud, S.; Jedlovszky, P. Adsorption of Acetaldehyde on Ice as Seen From Computer Simulation and Infrared Spectroscopy Measurements. Langmuir 2012, 28, 4198-4207.

(31) Mészár, Zs. E.; Hantal, Gy.; Picaud, S.; Jedlovszky, P. Adsorption of Aromatic Hydrocarbon Molecules at the Surface of Ice, As Seen by Grand Canonical Monte Carlo Simulation. J. Phys. Chem. C 2013, 117, 6719-6729. 
(32) Picaud, S.; Jedlovszky, P. Adsorption of $\mathrm{H}_{2} \mathrm{O}_{2}$ at the Surface of $\mathrm{I}_{\mathrm{h}}$ Ice, as Seen from Grand Canonical Monte Carlo Simulations. Chem. Phys. Letters 2014, 600, 73-78.

(33) Szőri, M.; Jedlovszky, P. Adsorption of HCN at the Surface of Ice. A Grand Canonical Monte Carlo Simulation Study. J. Phys. Chem. C 2014, 118, 3599-3609.

(34) Sumi, I.; Picaud, S.; Jedlovszky, P. Adsorption of Methylene Fluoride and Methylene Chloride at the Surface of Ice under Tropospheric Conditions. A Grand Canonical Monte Carlo Simulation Study. J. Phys. Chem. C 2015, 119, 17243-17252.

(35) Sumi, I.; Fábián, B.; Picaud, S.; Jedlovszky, P. Adsorption of Fluorinated Methane Derivatives at the Surface of Ice under Tropospheric Conditions, As Seen from Grand Canonical Monte Carlo Simulations. J. Phys. Chem. C 2016, 120, 17386-17399.

(36) Szentirmai, V.; Szőri, M.; Picaud, S.; Jedlovszky, P. Adsorption of Methylamine at the Surface of Ice. A Grand Canonical Monte Carlo Simulation Study J. Phys. Chem. C 2016, $120,23480-23489$.

(37) Metropolis, N.; Rosenbluth, A.W.; Rosenbluth, M.N.; Teller, A.H.; Teller, E. Equation of State Calculations by Fast Computing Machines. J. Chem. Phys. 1953, 21, 1087 - 1092.

(38) Mezei, M. A Cavity-Biased (T, V, $\mu$ ) Monte Carlo Method for the Computer Simulation of Fluids. Mol. Phys. 1980, 40, 901 - 906.

(39) Mezei, M. Grand-Canonical Ensemble Monte Carlo Study of Dense Liquid: LennardJones, Soft Spheres and Water. Mol. Phys. 1987, 61, 565 - 582. Erratum: 1989, 67, $1207-$ 1208.

(40) Daub, C. D.; Patey G. N.; Jack, D. B.; Sallabi A. K. Monte Carlo Simulations of the Adsorption of $\mathrm{CO}_{2}$ on the $\mathrm{MgO}(100)$ Surface. J. Chem. Phys. 2006, 124, 114706.

(41) NIST Standard Reference Database Number 69, NIST Chemistry Webbook, Ed., 2005, URL: http://webbook.nist.gov/chemistry/.

(42) Radhakrishnan, R.; Trout, B.L. A New Approach for Studying Nucleation Phenomena Using Molecular Simulations : Application to $\mathrm{CO}_{2}$ Hydrate Clathrates. J. Chem. Phys. 2002, 117, 1786-1796.

(43) Báez, L.A.; Clancy P. Computer Simulation of the Crystal Growth and Dissolution of Natural Gas Hydrates. Ann. N. Y. Acad. Sci., 1994, 715, 177-186

(44) Kerns, R.C.; Allen, L.C. Cyclic and Bifurcated Hydrogen Bonds. J. Am. Chem. Soc. 1978, 100, 6587-6594.

(45) Yeo, G.A.; Ford, T.A. Ab Initio Molecular Orbital Calculations of the Infrared Spectra of Hydrogen Bonded Complexes of Water, Ammonia, and Hydroxylamine. Part 6. The Infrared Spectrum of the Water-Ammonia Complex. Can. J. Chem. 1991, 69, 632-637.

(46) Xantheas, S.S. Intermolecular Interactions and Cooperative Effects from Electronic Structure Calculations: an Effective Means for Developing Interaction Potentials for Condensed Phase Simulations. in Novel Approaches to the Structure and Dynamics of Liquids: Experiments, Theories and Simulations. J. Samios and V.A. Durov. Kluwer Academic Publishers (Dordrecht, 2004). p. 1-15 
(47) Jedlovszky, P.; Pártay, L. B., Bartók, A. P.; Garberoglio, G.; Vallauri, R. Structure of Coexisting Liquid Phases of Supercooled Water: Analogy with Ice Polymorphs. J. Phys. Chem. 2007, 126, 241103-1-4.

(48) Jedlovszky, P.; Pártay, L. B., Bartók, A. P.; Voloshin, V. P.; Medvedev, N. N.; Garberoglio, G.; Vallauri, R. Structural and Thermodynamic Properties of Different Phases of Supercooled Water. J. Phys. Chem. 2008, 128, 244503-1-12.

(49) Handle, P.H.; Loerting, T.; Sciortino, F. Supercooled and Glassy Water: Metastable Liquid(s), Amorphous solid(s), and a No-Man's Land. PNAS 2017, 114 (51), 1333613344.

(50) Brodholt, J.; Sampoli, M.; Vallauri, R. Parametrizing a Polarizable Intermolecular Potential for Water. Mol. Phys. 1995, 86, 149-158.

(51) Martelli, F.; Torquato, S.; Giovambattista, N.; Car R. Large-Scale Structure and Hyperuniformity of Amorphous Ices. Phys. Rev. Lett. 2017, 119, 136002

(52) Jedlovszky, P.; Vallauri, R. Liquid-Vapor and Liquid-Liquid Phase Equilibria of the Brodholt-Sampoli-Vallauri Polarizable Water Model. J. Chem. Phys. 2005, 122, 081101$1-4$.

(53) Gallo, P.; Amman-Winkel, K.; Angell, C.A., Anisimov, M.; Caupin, F.; Chakravarty, C.; Lascaris, E.; Loerthing, T.; Panagiotopoulos, A.; Russo, J.; Sellberg, J.A.; Stanley, H.E.; Anaka, H.; Vega, C.; Xu, L.; Pettersson, L.G.M. Water: A Tale of Two Liquids. Chem. Rev. 2016, 116, 7463-7500.

(54) Engstler, J.; Giovambattista, N. Heating- and Pressure-Induced Transformations in Amorphous and Hexagonal Ice: A Computer Simulation Study Using the TIP4P/2005 Model. J. Chem. Phys. 2017, 147, 074505.

(55) Petuya, C.; Damay, F.; Chazallon, B.; Bruneel J.L.; Desmedt, A. Guest Partitioning and Metastability of the Nitrogen Gas Hydrate. J. Phys. Chem. C 2018, 122, 566-573.

(56) Falenty, A.; Hansen, T.C.; Kuhs, W.F. Formation and Properties of Ice XVI Obtained by Emptying a Type sII Clathrate Hydrate. Nature 2014, 516, 231.

(57) McLaurin, G.; Shin, K.; Alavi, S.; Ripmeester, J.A. Antifreezes Act a Catalysts for Methane Hydrate Formation from Ice. Angew. Chem., Int. Ed. 2014, 53, 10429-10433.

(58) Mousis, O.; Chassefière, E.; Holm ; N.G. ; Bouquet, A. ; Waite, J.H. ; Geppert, W.D. ; Picaud, S. ; Aikawa, Y.; Ali-Dib, M.; Charlou, J.L.; Rousselot, P. Methane Clathrates in the Solar System. Astrobiology 2015, 15, 308-326.

(59) Vu, T.H.; Gloesener, E.; Choukroun, M. ; Ibourichene, A. ; Hodyss, R. Experimental Study on the Effect of Ammonia on the Phase Behavior of Tetrahydrofuran Clathrates. $J$. Phys. Chem. B 2014, 118, 13371-13377.

(60) Tobie, G.; Lunine, J.I.; Sotin, C. Episodic Outgassing as the Origin of Atmospheric Methane on Titan. Nature, 2006, 440, 61-64.

(61) Spencer, J.R.; Tamppari, L.K.; Martin, T.Z.; Travis, L.D. Temperature on Europa from Galileo Photopolarimeter-Radiometer: Nighttime Thermal Anomalies. Science 1999, 284, 1514. 
Table 1. Interaction Parameters and geometry of the Molecular Models Used ${ }^{\mathrm{a}}$

\begin{tabular}{ccccc}
\hline \hline Molecule & & & & \\
\hline $\mathrm{NH}_{3}{ }^{\mathrm{b}}$ & Site & $\sigma / \AA$ & $\varepsilon / \mathrm{kJ} \mathrm{mol}^{-1}$ & $q / \mathrm{e}$ \\
$\mathrm{N}$ & 3.42 & 0.7113 & -1.020 \\
$\mathrm{H}$ & & & 0.340 \\
& bond & bond length $(\AA)$ & Angle & bond angle (deg) \\
& $\mathrm{N}-\mathrm{H}$ & 1.010 & $\mathrm{H}-\mathrm{N}-\mathrm{H}$ & 106.4 \\
\hline $\mathrm{H}_{2} \mathrm{O}^{\mathrm{c}}$ & $\mathrm{O}$ & 3.1668 & 0.8822 & 0.000 \\
& $\mathrm{H}$ & - & - & 0.5897 \\
& $\mathrm{M}^{\mathrm{d}}$ & - & - & -1.1794 \\
& bond & bond length $(\AA)$ & Angle & bond angle (deg) \\
& $\mathrm{O}-\mathrm{H}$ & 0.9572 & $\mathrm{H}-\mathrm{O}-\mathrm{H}$ & 104.52 \\
& $\mathrm{O}-\mathrm{M}$ & 0.1577 & & \\
\hline \hline
\end{tabular}

${ }^{\mathrm{a}} \sigma, \varepsilon$ and $q$ stand for the Lennard-Jones distance and energy parameters and for the fractional charges, respectively.

${ }^{\mathrm{b}}$ OPLS model [24]

${ }^{\mathrm{c}} \mathrm{TIP} 4 \mathrm{P} /$ Ice model [23]

${ }^{\mathrm{d}}$ Non-atomic interaction site 


\section{Figure legends}

Figure 1. Occupancy isotherm of $\mathrm{NH}_{3}$ in a (a) rigid (top panel) and (b) flexible (bottom panel) clathrate hydrate, as obtained from our GCMC simulations at $100 \mathrm{~K}$ (black squares), $150 \mathrm{~K}$ (red circles) and $180 \mathrm{~K}$ (green triangles). Filled and empty symbols correspond to $2 \times 2 \times 2$ and $3 \times 3 \times 3$ clathrate units in the basic box. The insets show the occupancy isotherms calculated as a function of the relative pressure $\mathrm{p}_{\mathrm{rel}}=\mathrm{p} / \mathrm{p}_{0}$. The lines connecting the points are just guides to the eye. The arrows indicate the chemical potential values corresponding to the point of condensation of $\mathrm{NH}_{3}$. Note that, for the ordinate axis, we divide the number of ammonia molecules in the simulation box by the number of unit cells of the rigid clathrate, as an arbitrary normalization.

Figure 2. Some snapshots of the systems in a flexible clathrate hydrate of $\mathrm{NH}_{3}$, as obtained from our GCMC simulations at $100 \mathrm{~K}$ at (a) low $(-21.72 \mathrm{~kJ} / \mathrm{mol})$ and (b) high $(-10.08 \mathrm{~kJ} / \mathrm{mol})$ chemical potential values corresponding to different fillings of the cages. $\mathrm{O}, \mathrm{N}$, and $\mathrm{H}$ atoms are shown by red, blue and white balls, respectively. Note that snapshots for the same chemical potential values are given on the right hand side of the figure, in which ammonia molecules have been removed for clarity (i.e., only the water network is shown)

Figure 3. Tetrahedral order parameter (red squares) calculated for the flexible $\mathrm{NH}_{3}$ clathrates, as a function of the chemical potential, at $100 \mathrm{~K}$ (top panel), $150 \mathrm{~K}$ (middle panel), and $180 \mathrm{~K}$ (bottom panel). The corresponding occupancy isotherms are also shown (black circles). The lines connecting the points are just guides to the eye. The dashed vertical lines indicate the chemical potential values corresponding to the point of condensation of $\mathrm{NH}_{3}$. Scales at the left and right side of the figure correspond to the occupancy isotherm and order parameter data, respectively. Note that, for the ordinate axis, we divide the number of ammonia molecules in the simulation box by the number of unit cells of the rigid clathrate, as an arbitrary normalization.

Figure 4. Distributions of the tetrahedral order parameter, obtained by considering only the $\mathrm{O}$ atoms, for various chemical potential values, corresponding to different $\mathrm{NH}_{3}$ loadings, in the flexible clathrate at $100 \mathrm{~K}$ (top panel), $150 \mathrm{~K}$ (middle panel), and $180 \mathrm{~K}$ (bottom panel). The 
insets show the region of the peak around 0.4 on a magnified scale. The percentage of atoms giving rise to this peak is also indicated.

Figure 5. Distribution of the tetrahedral order parameter, obtained by considering both the $\mathrm{O}$ and $\mathrm{N}$ atoms, for various chemical potential values, corresponding to different $\mathrm{NH}_{3}$ loadings, in the flexible clathrate at $100 \mathrm{~K}$ (top panel), $150 \mathrm{~K}$ (middle panel) and $180 \mathrm{~K}$ (bottom panel).

Figure 6. Fraction of water molecules that belong to a given number of cyclic pentamers in the systems simulated at (a) $100 \mathrm{~K}$, (b) $150 \mathrm{~K}$ and (c) $180 \mathrm{~K}$ at various chemical potential values, corresponding to different $\mathrm{NH}_{3}$ loadings. The distributions corresponding to the perfect rigid clathrate and to the perfect $I_{h}$ ice crystal are given as a reference in the top and bottom panels, respectively.

Figure 7. Partial radial distribution functions between the $O$ atoms of the water cages (top panels), between the $\mathrm{O}$ and $\mathrm{N}$ atoms (middle panels), and between the $\mathrm{N}$ atoms (bottom panels), as obtained at (a) $100 \mathrm{~K}$, (b) $150 \mathrm{~K}$ and (c) $180 \mathrm{~K}$ at various chemical potential values, corresponding to different $\mathrm{NH}_{3}$ loadings. The radial distribution functions calculated for the rigid clathrate at full occupancy is also given as a reference. The curves corresponding to increasing chemical values are shifted up by 2, 4, 6, 8, and 10 units, for clarity. 
Figure 1.

Fábián et al.

(a)

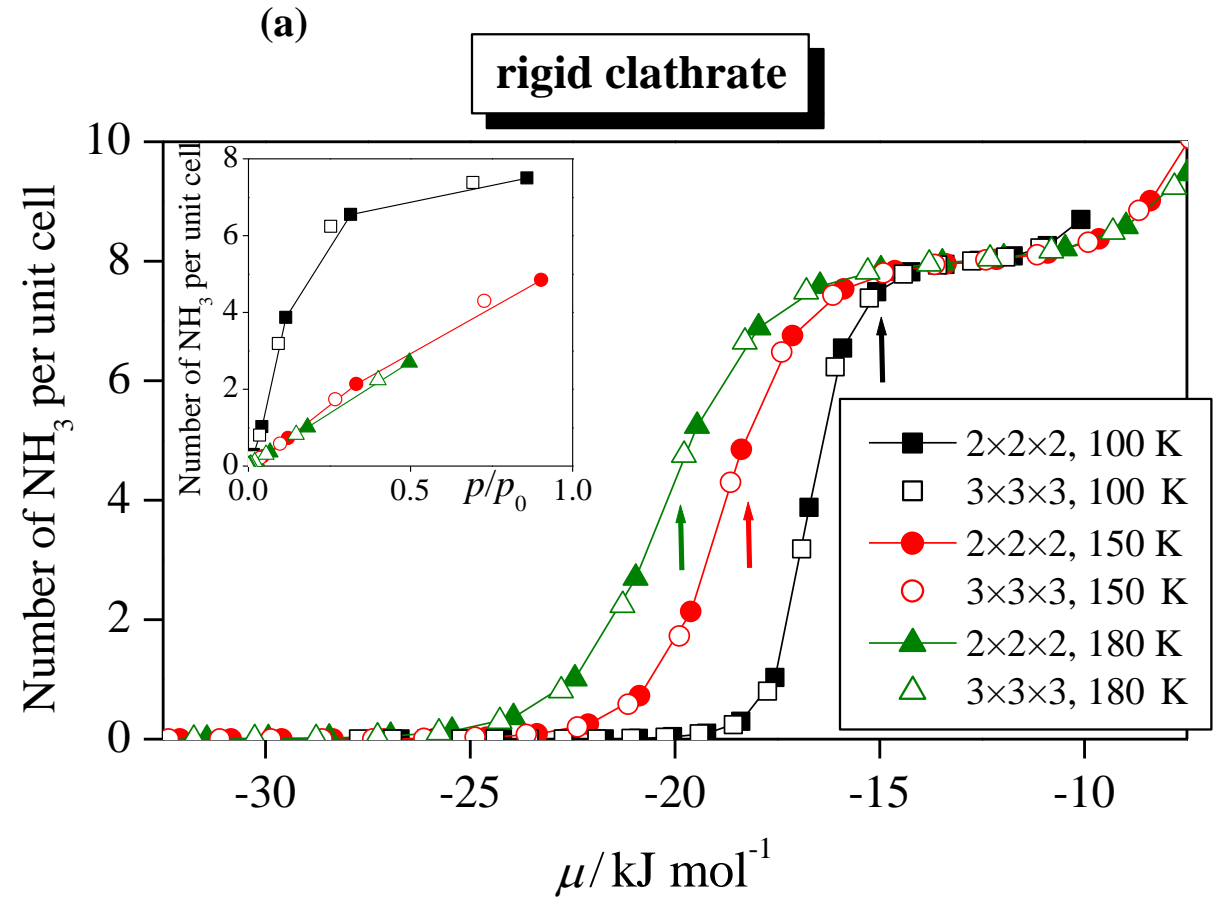

(b)

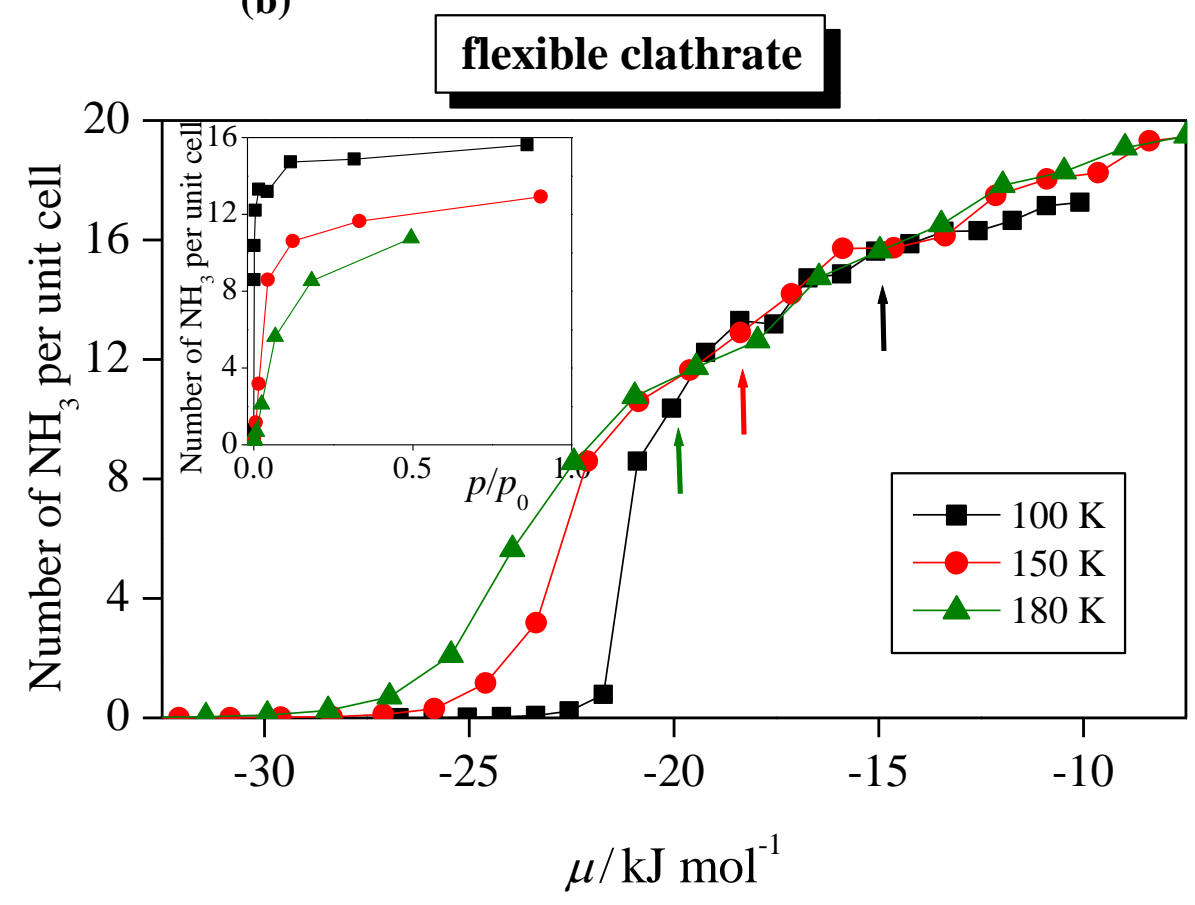


Figure 2.

Fábián et al.

(a)
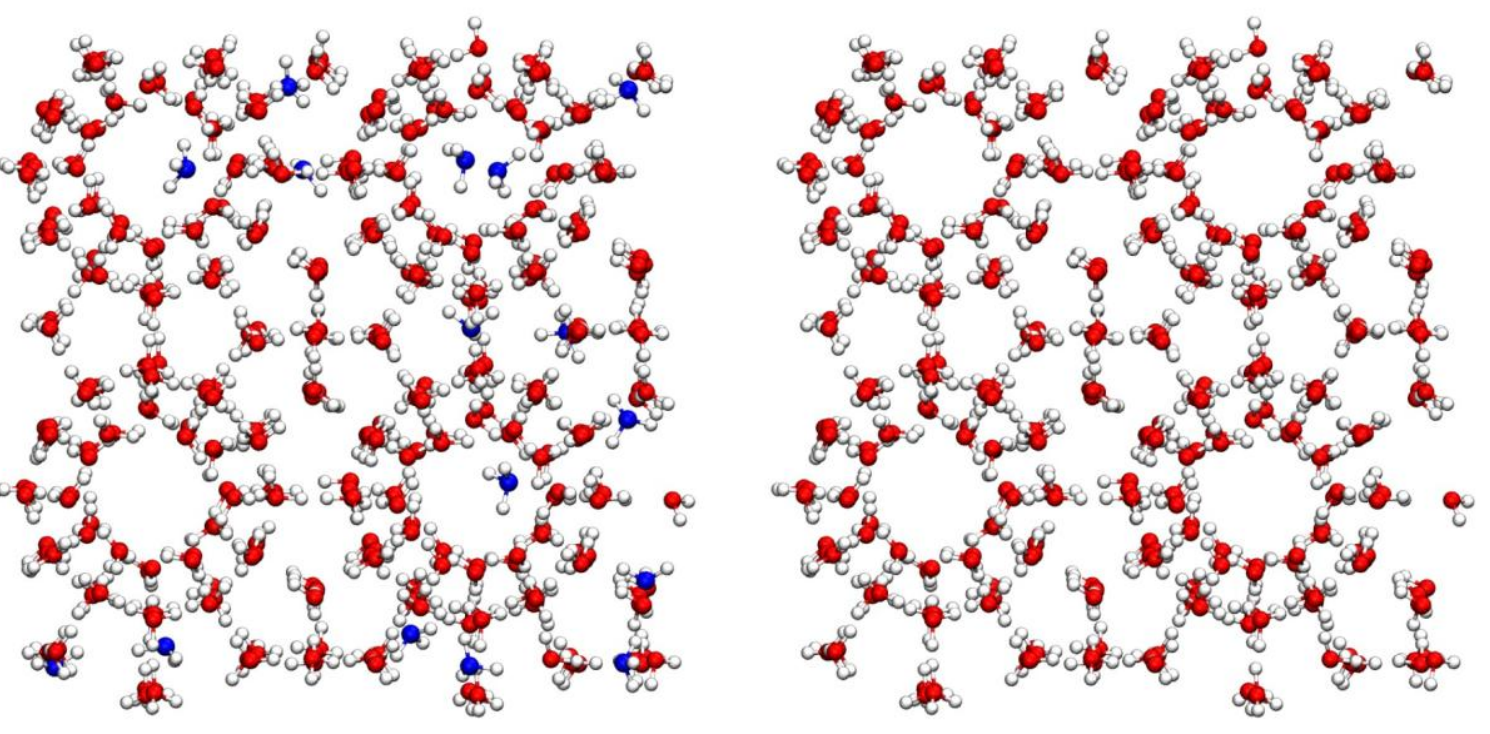

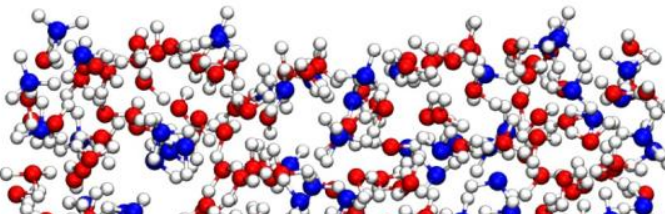

(b)
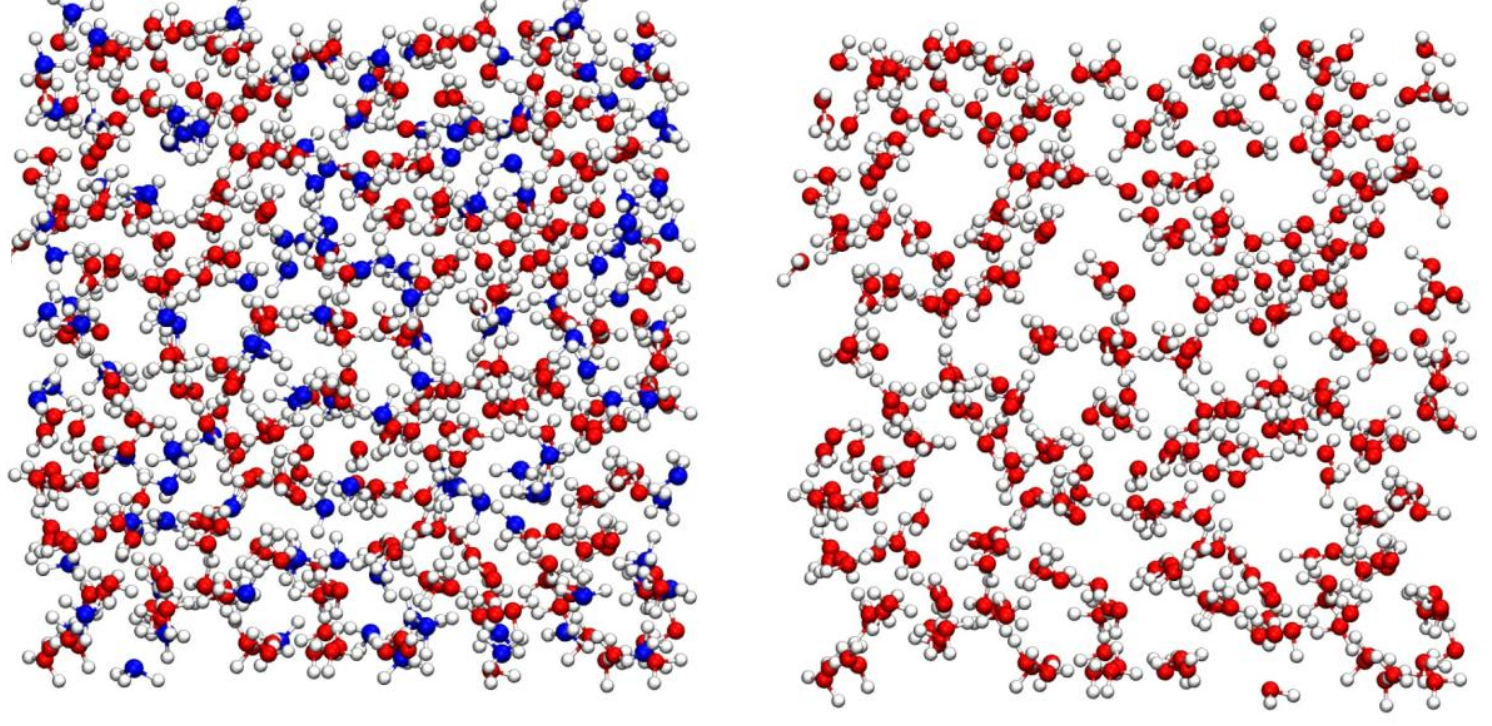
Figure 3.

Fábián et al.

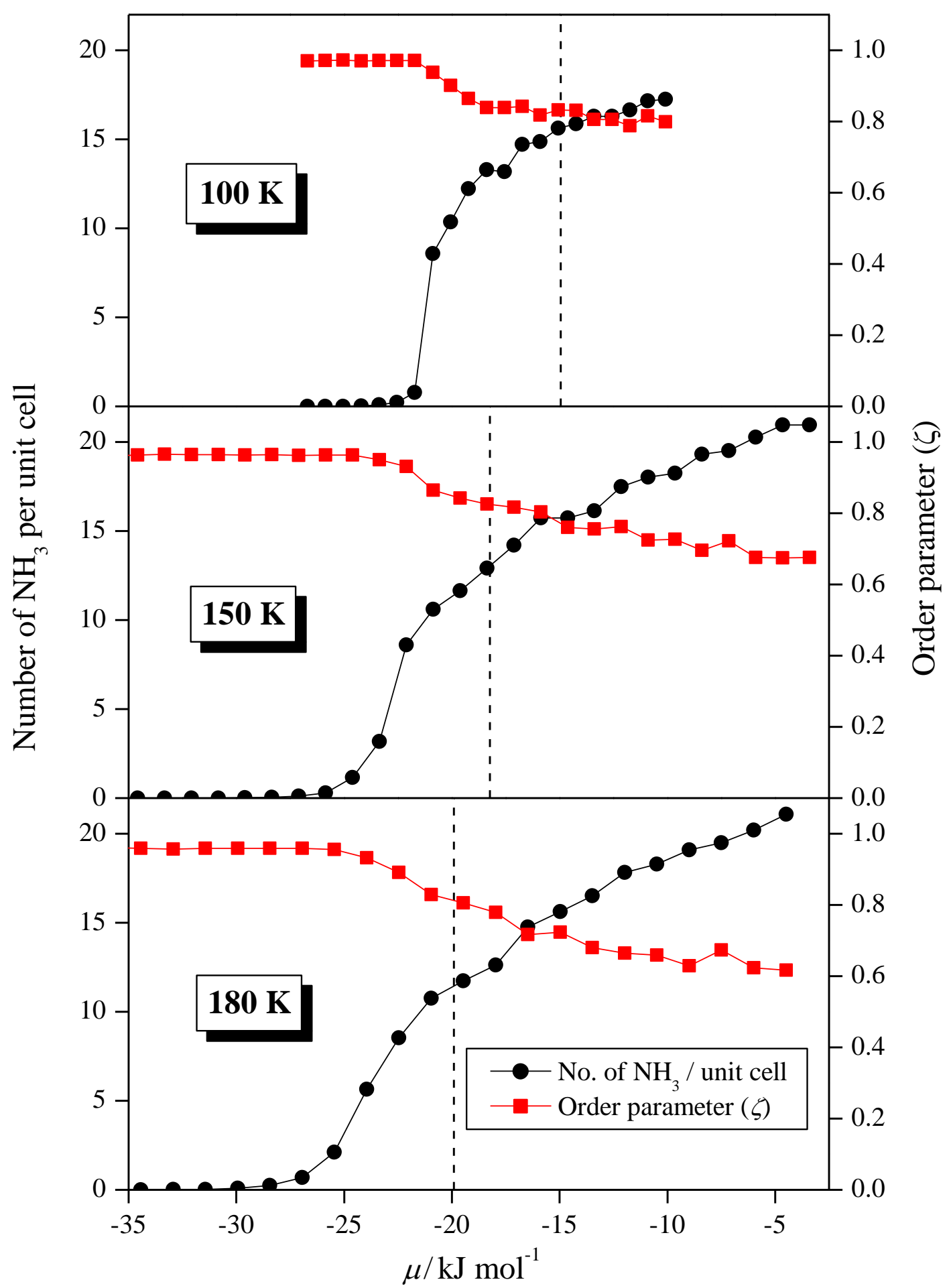


Figure 4.

Fábián et al.

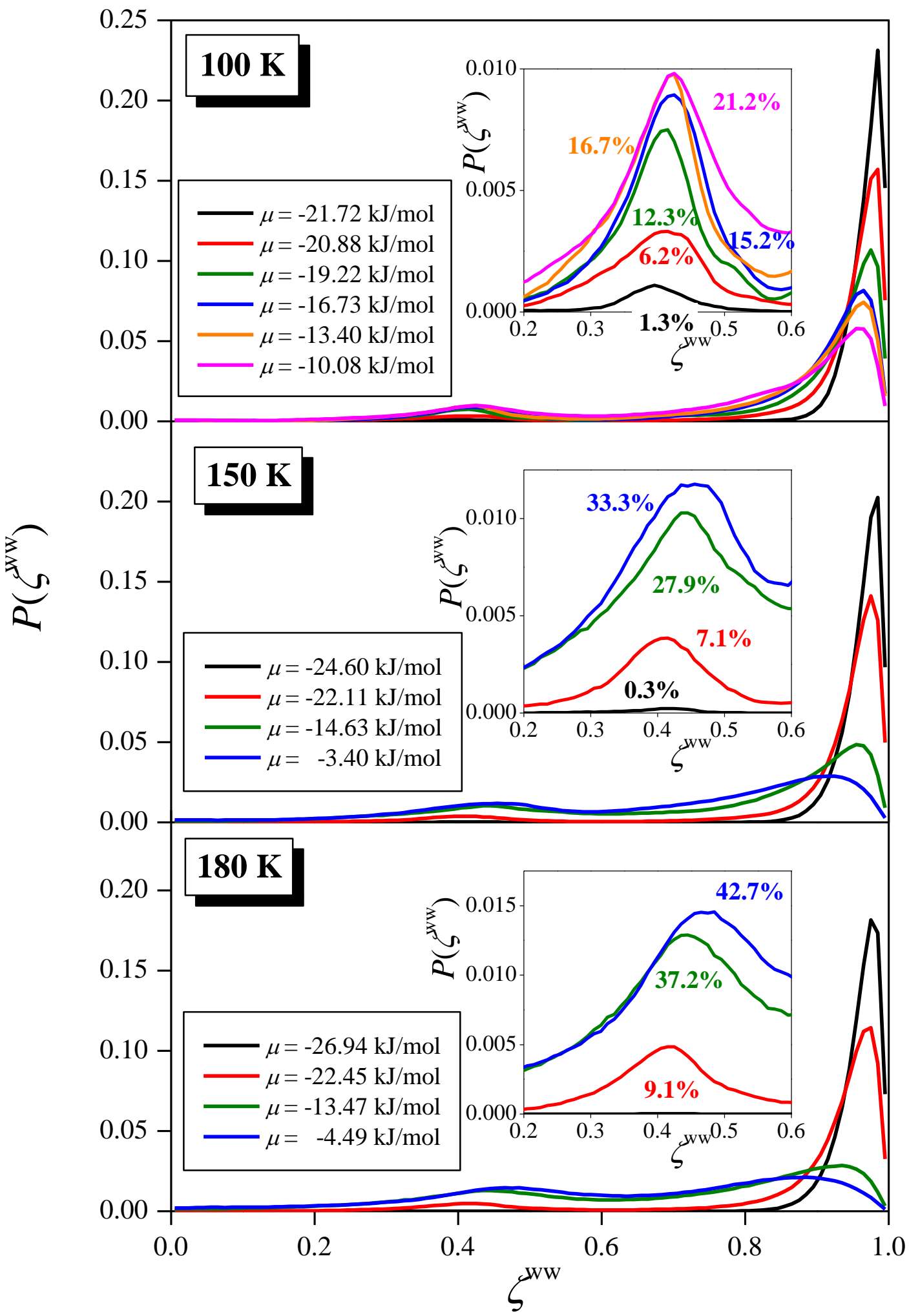


Figure 5.

Fábián et al.

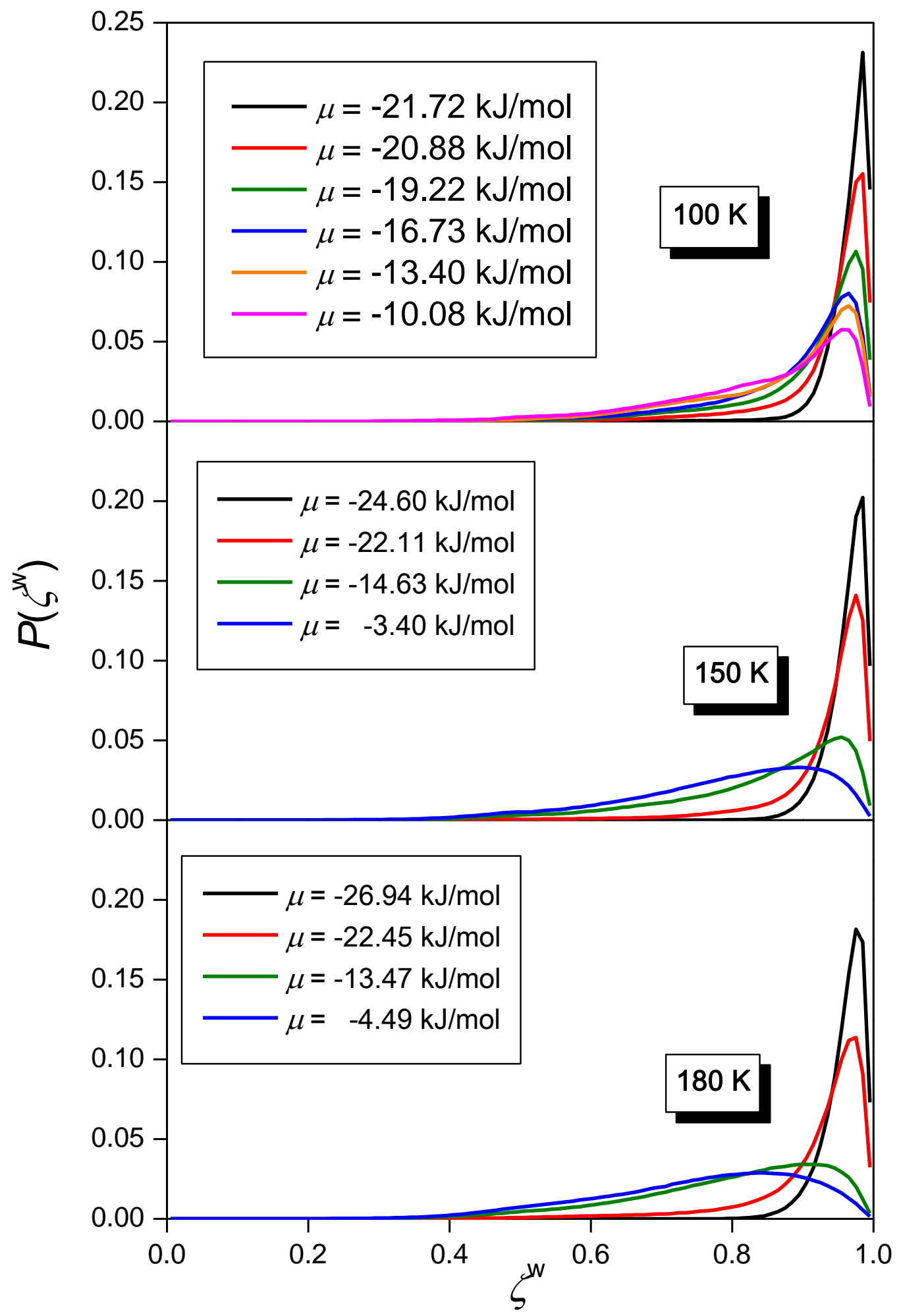


Figure 6a.

Fábián et al.

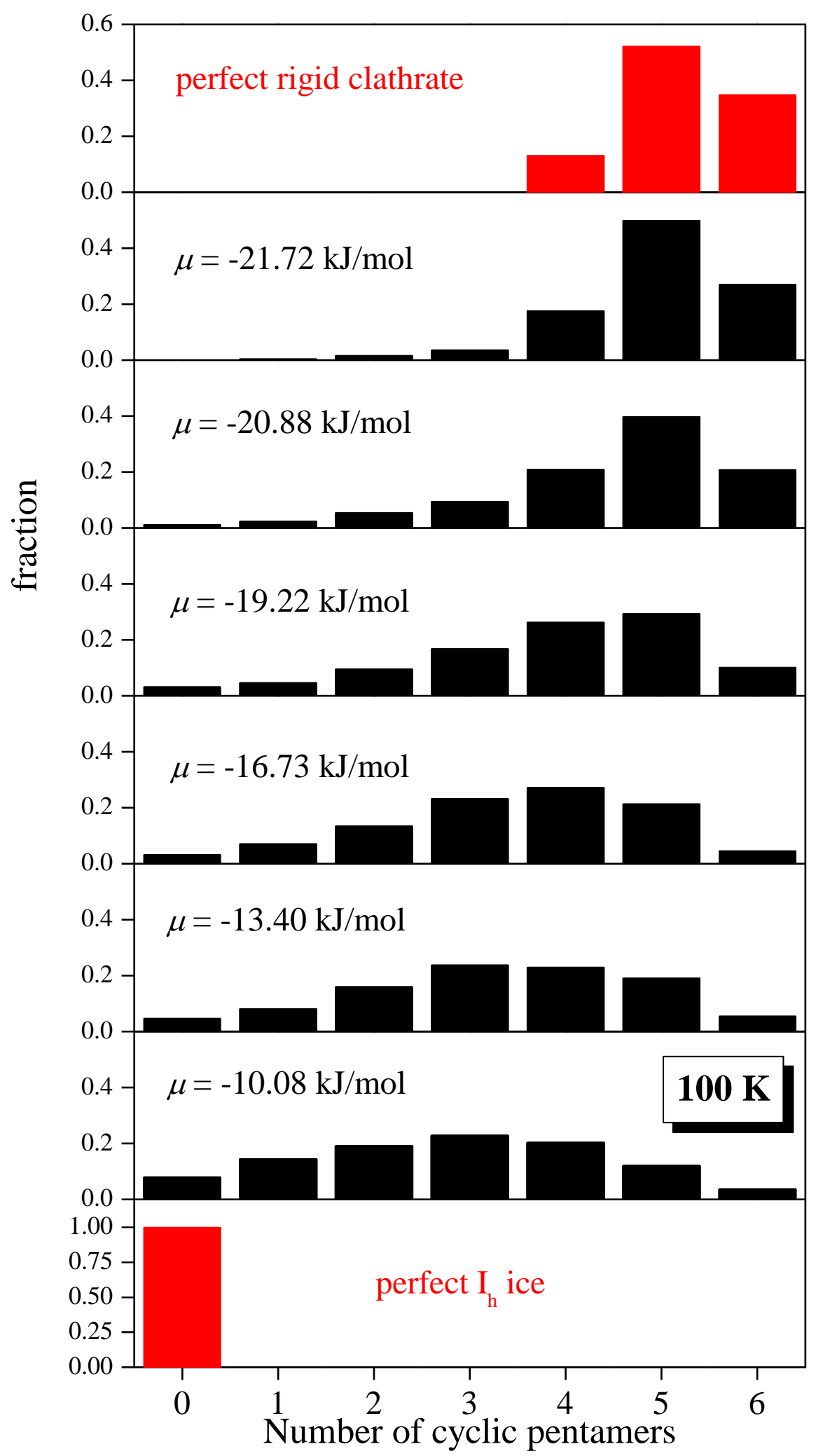


Figure 6b.

Fábián et al.

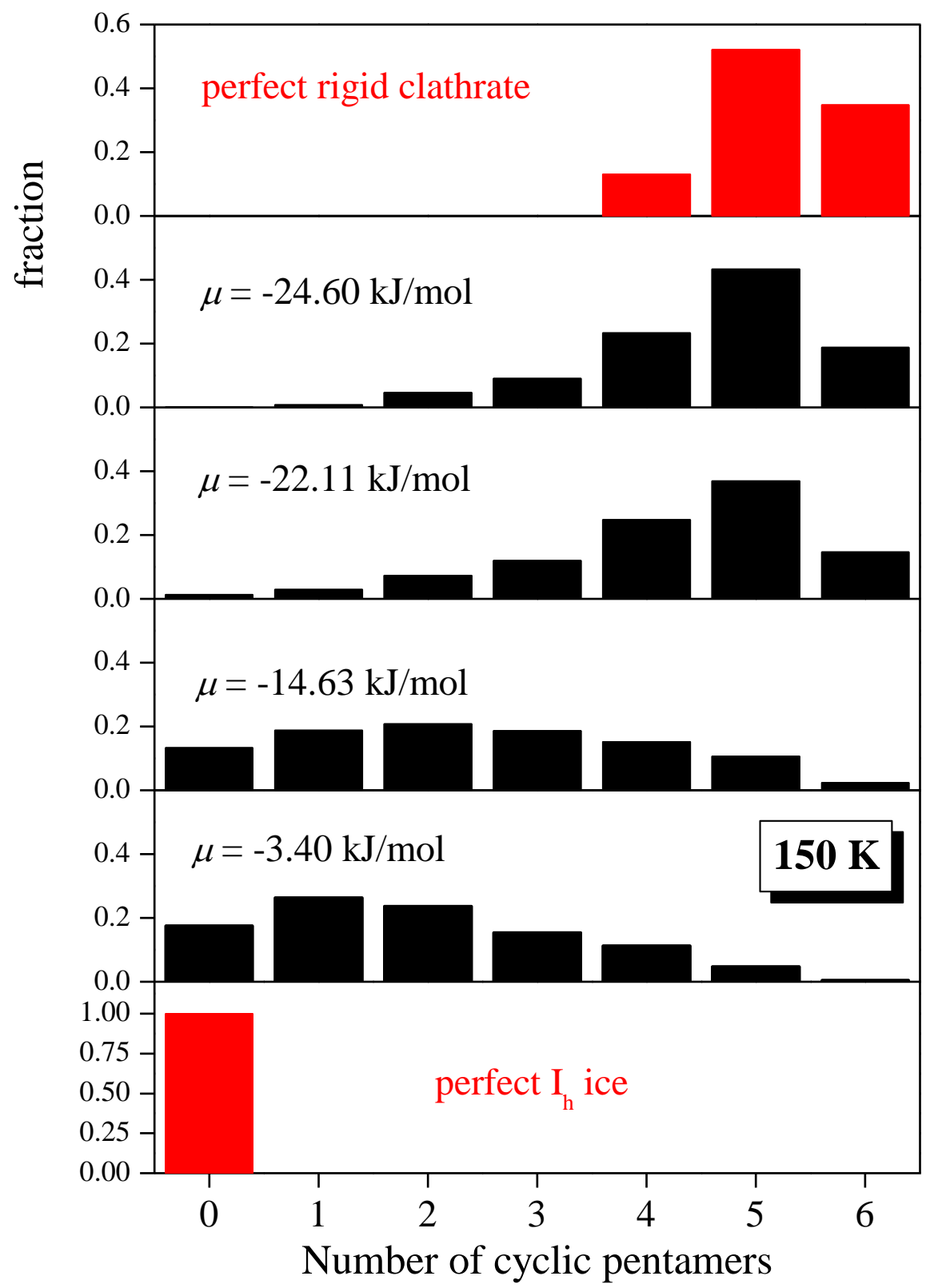


Figure 6c.

Fábián et al.

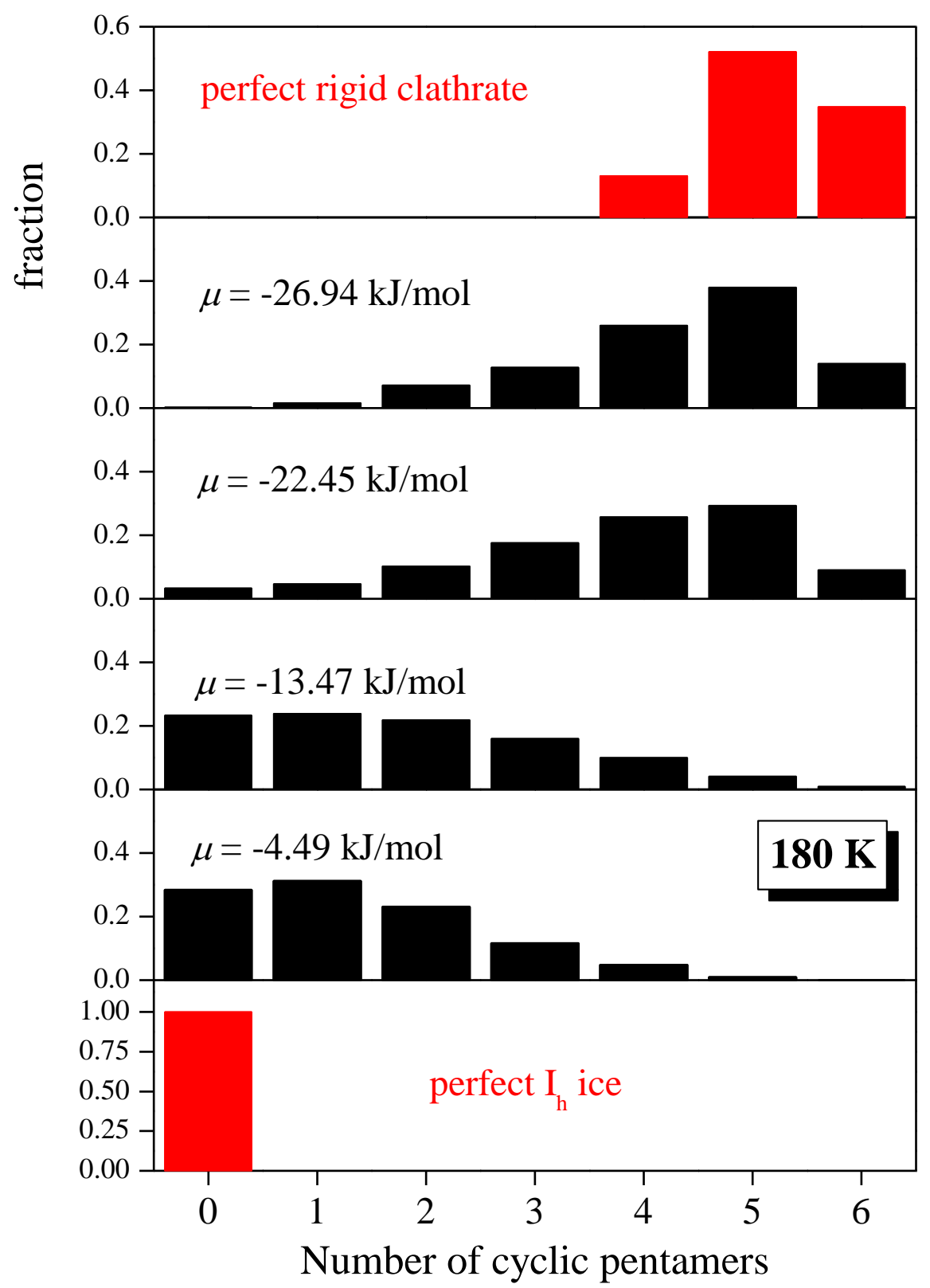


Figure 7a.

Fábián et al.

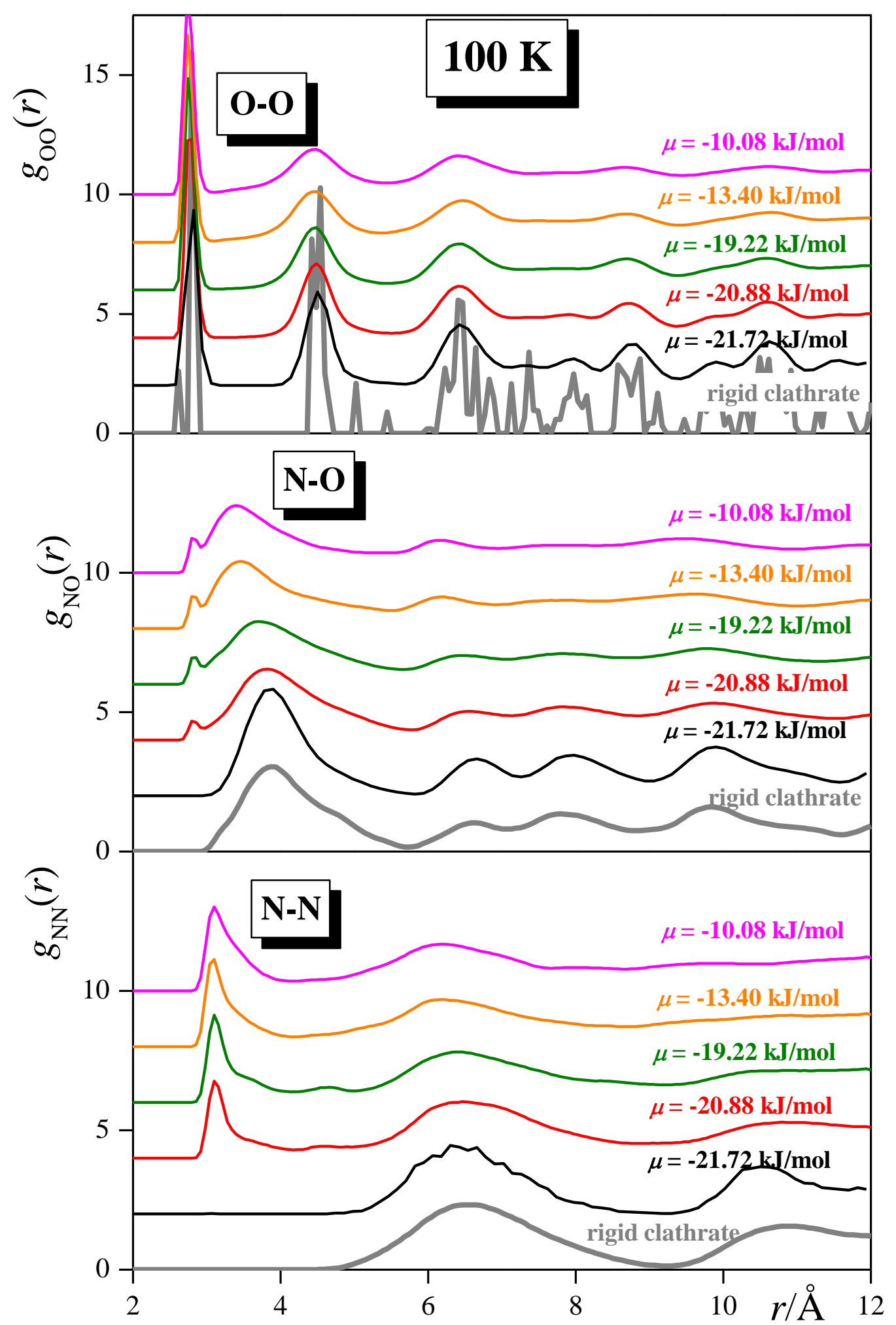


Figure 7b.

Fábián et al.

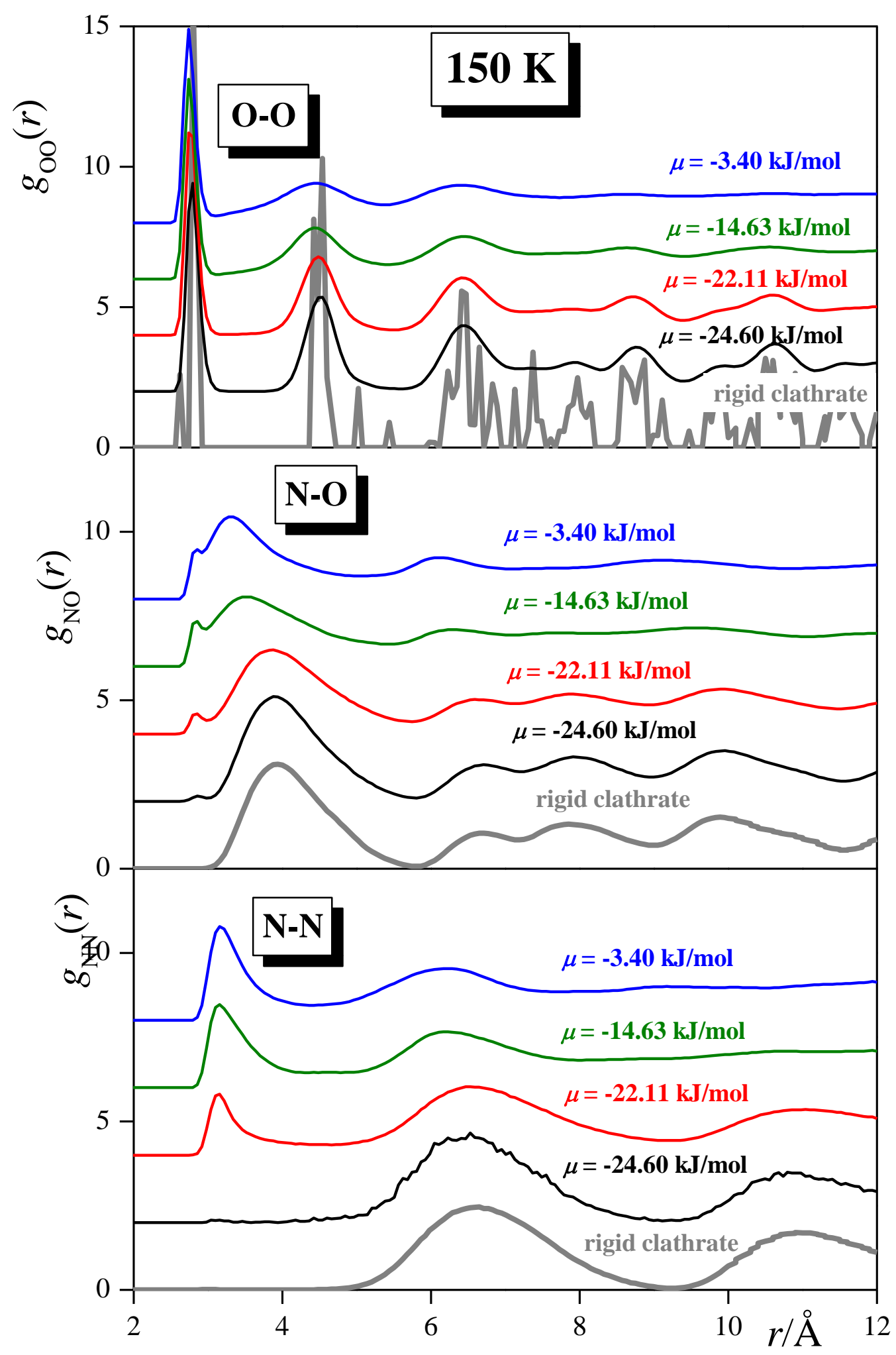


Figure 7c.

Fábián et al.

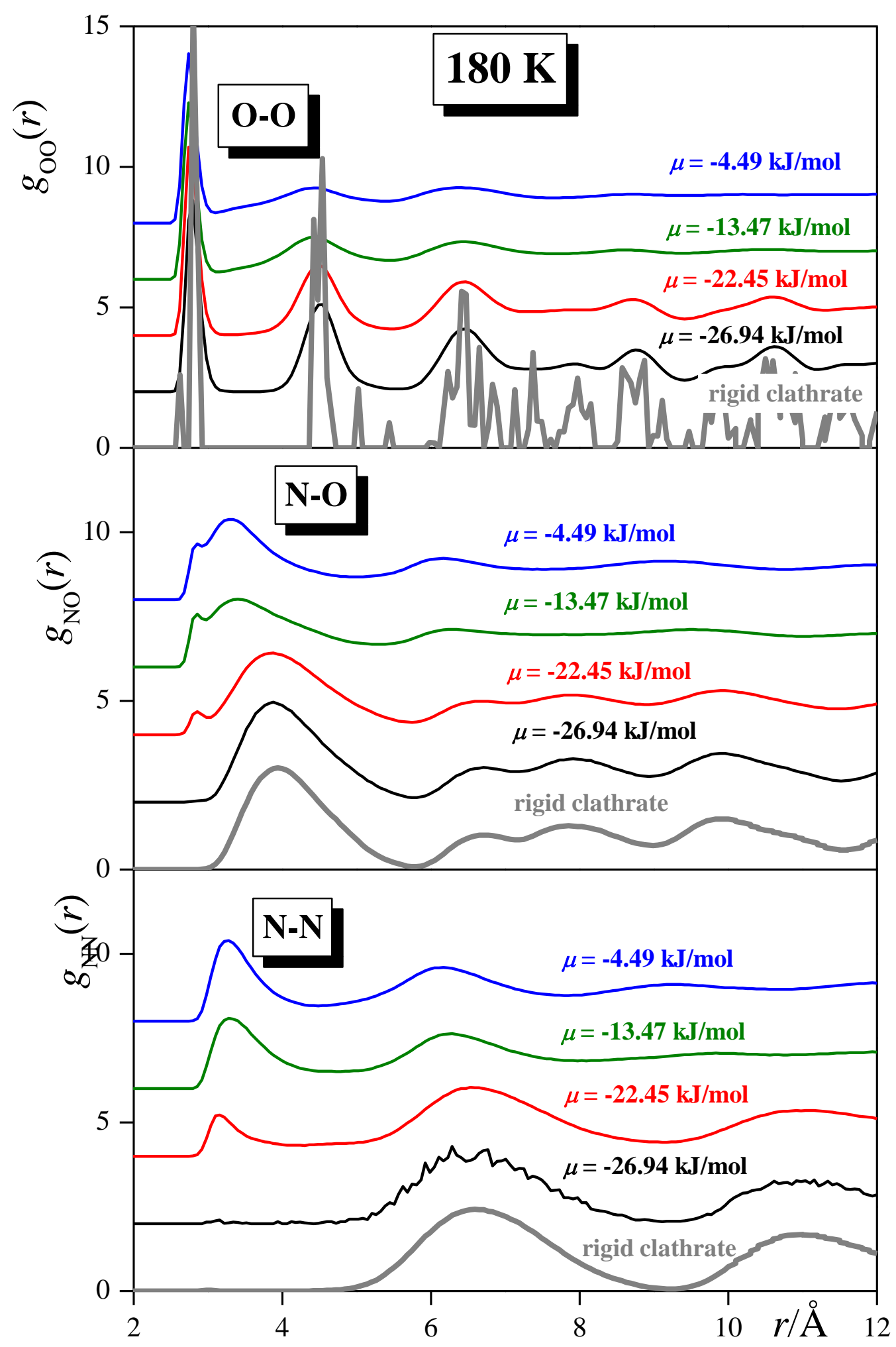


Table of Contents Graphics:

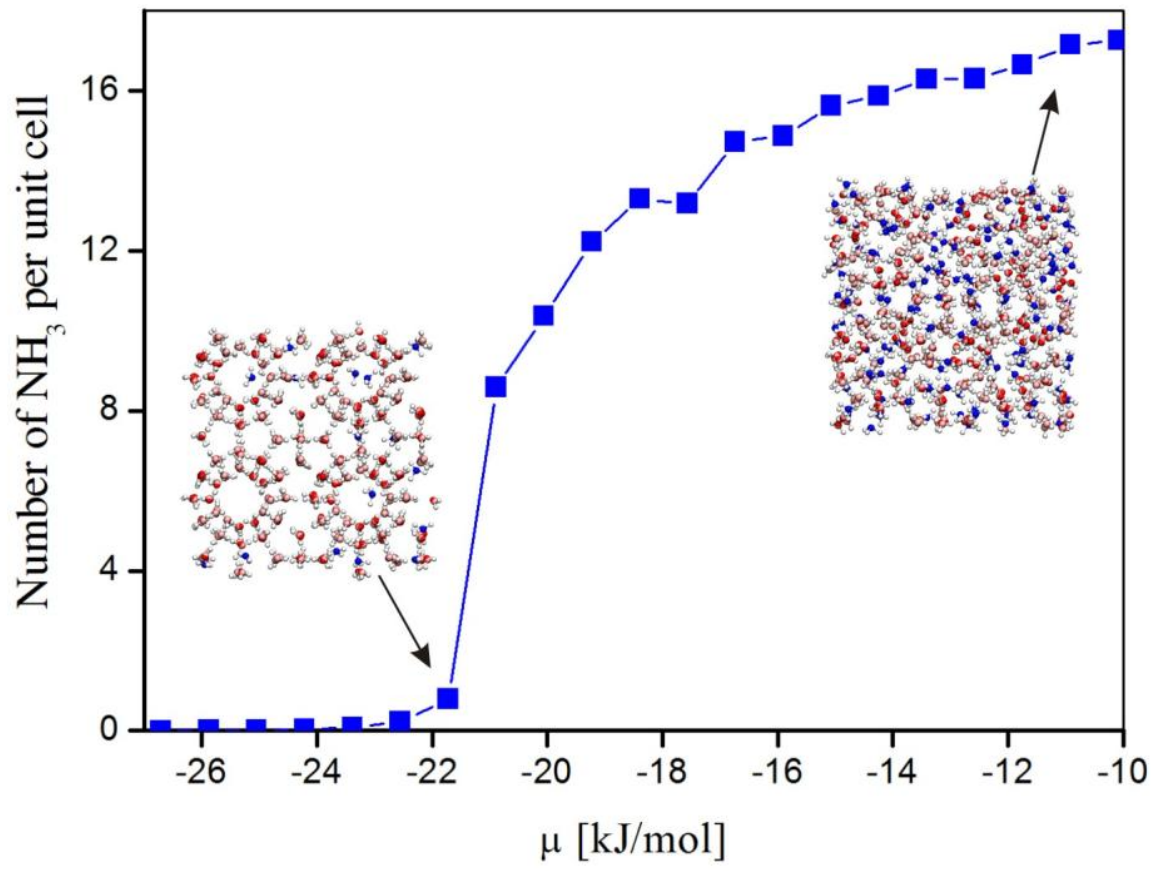

